\title{
Real-time Electron Dynamics Study of Plasmon-mediated Photocatalysis on an Icosahedral $\mathrm{Al}_{13}{ }^{-1}$ Nanocluster
}

Pratima Pandeya ${ }^{\mathrm{a}}$ and Christine M. Aikens ${ }^{\mathrm{a},{ }^{*}}$

a) Department of Chemistry, Kansas State University, Manhattan, KS 66506, USA

* cmaikens@ksu.edu; 1-785-532-0954

Table S1. Coordinates for the optimized $\mathrm{Al}_{13}{ }^{-1}$ cluster:

$\begin{array}{rrrr}\text { Al } & 0.000000 & 0.000000 & 0.000000 \\ \text { Al } & 2.627919 & -0.000000 & -0.000000 \\ \text { Al } & 1.174943 & 2.350519 & 0.000000 \\ \text { Al } & 1.174943 & 0.726350 & 2.235477 \\ \text { Al } & 1.174943 & -1.901610 & 1.381600 \\ \text { Al } & 1.174943 & -1.901610 & -1.381600 \\ \text { Al } & 1.174943 & 0.726350 & -2.235477 \\ \text { Al } & -1.174943 & 1.901610 & -1.381600 \\ \text { Al } & -1.174943 & 1.901610 & 1.381600 \\ \text { Al } & -1.174943 & -0.726350 & 2.235477 \\ \text { Al } & -1.174943 & -2.350519 & 0.000000 \\ \text { Al } & -1.174943 & -0.726350 & -2.235477 \\ \text { Al } & -2.627919 & 0.000000 & -0.000000\end{array}$

Table S2. Coordinates for the optimized $\left[\mathrm{Al}_{13} \mathrm{~N}_{2}\right]^{-1}$ cluster:

$\begin{array}{lrrr}\text { Al } & 0.488829 & -0.000001 & 0.000001 \\ \text { Al } & -0.901510 & 0.006595 & 2.230322 \\ \text { Al } & 0.492878 & 2.239647 & 1.374889 \\ \text { Al } & -1.743594 & 1.386401 & -0.003893 \\ \text { Al } & -1.743578 & -1.386428 & 0.004321 \\ \text { Al } & 0.492917 & -2.231467 & 1.388097 \\ \text { Al } & 1.861571 & 0.006664 & 2.240452 \\ \text { Al } & 2.727053 & 1.376380 & -0.004316 \\ \text { Al } & 0.492617 & 2.231469 & -1.388092 \\ \text { Al } & -0.901939 & -0.006614 & -2.230056 \\ \text { Al } & 0.492643 & -2.239651 & -1.374882 \\ \text { Al } & 2.727074 & -1.376347 & 0.003877 \\ \text { Al } & 1.861131 & -0.006645 & -2.240722 \\ \mathrm{~N} & -5.892812 & 0.549900 & -0.000276 \\ \mathrm{~N} & -5.892786 & -0.549902 & 0.000277\end{array}$


Table S3. Coordinates for the optimized $\left[\mathrm{Al}_{13} \mathrm{~N}_{2}\right]^{-1}$ cluster with origin at center of mass:

$\begin{array}{lrrr}\text { Al } & 0.47247928 & -0.00000114 & 0.00000111 \\ \text { Al } & -0.91785972 & 0.00659486 & 2.23032211 \\ \text { Al } & 0.47652828 & 2.23964686 & 1.37488911 \\ \text { Al } & -1.75994372 & 1.38640086 & -0.00389289 \\ \text { Al } & -1.75992772 & -1.38642814 & 0.00432111 \\ \text { Al } & 0.47656728 & -2.23146714 & 1.38809711 \\ \text { Al } & 1.84522128 & 0.00666386 & 2.24045211 \\ \text { Al } & 2.71070328 & 1.37637986 & -0.00431589 \\ \text { Al } & 0.47626728 & 2.23146886 & -1.38809189 \\ \text { Al } & -0.91828872 & -0.00661414 & -2.23005589 \\ \text { Al } & 0.47629328 & -2.23965114 & -1.37488189 \\ \text { Al } & 2.71072428 & -1.37634714 & 0.00387711 \\ \text { Al } & 1.84478128 & -0.00664514 & -2.24072189 \\ \text { N } & -5.90916172 & 0.54989986 & -0.00027589 \\ \text { N } & -5.90913572 & -0.54990214 & 0.00027711\end{array}$


Table S4. Transitions for the $\left[\mathrm{Al}_{13} \mathrm{~N}_{2}\right]^{-1}$ system with the highest oscillator strengths for the 7 $\mathrm{eV}$ plasmon peak from the LR-TDDFT calculation

\begin{tabular}{|c|c|c|c|c|c|}
\hline $\begin{array}{l}\text { Excited } \\
\text { States }\end{array}$ & Energy $(\mathrm{eV})$ & $\begin{array}{l}\text { Oscillator } \\
\text { Strength }\end{array}$ & Weight & Transitions & Orbitals \\
\hline 218 & 6.9429 & 1.2839 & $\begin{array}{c}0.23646 \\
0.14799 \\
-0.16659 \\
0.20255 \\
-0.10132 \\
-0.10088 \\
0.30276 \\
0.10196 \\
0.12760 \\
-0.18630 \\
-0.11116\end{array}$ & $\begin{array}{c}78 \rightarrow 96 \\
80 \rightarrow 94 \\
81 \rightarrow 95 \\
87 \rightarrow 115 \\
88 \rightarrow 114 \\
88 \rightarrow 118 \\
89 \rightarrow 113 \\
89 \rightarrow 116 \\
90 \rightarrow 122 \\
92 \rightarrow 111 \\
92 \rightarrow 117\end{array}$ & $\begin{array}{l}1 \mathrm{D} \rightarrow 1 \mathrm{G} \\
1 \mathrm{D} \rightarrow 1 \mathrm{G} \\
1 \mathrm{D} \rightarrow 1 \mathrm{G} \\
1 \mathrm{~F} \rightarrow 2 \mathrm{~F} \\
1 \mathrm{~F} \rightarrow 2 \mathrm{~F} \\
1 \mathrm{~F} \rightarrow 2 \mathrm{~F} \\
1 \mathrm{~F} \rightarrow 2 \mathrm{~F} \\
1 \mathrm{~F} \rightarrow 2 \mathrm{~F} \\
1 \mathrm{~F} \rightarrow 1 \mathrm{H} \\
1 \mathrm{~F} \rightarrow 3 \mathrm{P} \\
1 \mathrm{~F} \rightarrow 2 \mathrm{~F}\end{array}$ \\
\hline 220 & 6.9483 & 1.0050 & $\begin{array}{c}0.20787 \\
-0.17846 \\
-0.15903 \\
-0.10356 \\
-0.15355 \\
0.37828 \\
0.11446 \\
0.10714 \\
-0.13142\end{array}$ & $\begin{array}{c}77 \rightarrow 94 \\
78 \rightarrow 97 \\
84 \rightarrow 109 \\
86 \rightarrow 112 \\
86 \rightarrow 114 \\
87 \rightarrow 113 \\
90 \rightarrow 120 \\
91 \rightarrow 112 \\
92 \rightarrow 110\end{array}$ & $\begin{array}{l}1 \mathrm{D} \rightarrow 1 \mathrm{G} \\
1 \mathrm{D} \rightarrow 1 \mathrm{G} \\
2 \mathrm{P} \rightarrow 2 \pi^{*} \\
1 \mathrm{~F} \rightarrow 3 \mathrm{P} \\
1 \mathrm{~F} \rightarrow 2 \mathrm{~F} \\
1 \mathrm{~F} \rightarrow 2 \mathrm{~F} \\
1 \mathrm{~F} \rightarrow 1 \mathrm{H} \\
1 \mathrm{~F} \rightarrow 3 \mathrm{P} \\
1 \mathrm{~F} \rightarrow 3 \mathrm{P}\end{array}$ \\
\hline 221 & 6.9487 & 4.7267 & $\begin{array}{c}0.10826 \\
-0.11315 \\
0.13206 \\
0.29849 \\
0.10118 \\
0.12902 \\
-0.12504 \\
0.12648 \\
0.16748 \\
0.12595 \\
0.14374 \\
-0.12653 \\
0.12356 \\
-0.11294 \\
0.12354 \\
-0.14139\end{array}$ & $\begin{array}{c}77 \rightarrow 94 \\
83 \rightarrow 104 \\
84 \rightarrow 102 \\
84 \rightarrow 109 \\
85 \rightarrow 103 \\
86 \rightarrow 101 \\
86 \rightarrow 104 \\
87 \rightarrow 100 \\
87 \rightarrow 113 \\
88 \rightarrow 99 \\
88 \rightarrow 102 \\
89 \rightarrow 98 \\
89 \rightarrow 105 \\
90 \rightarrow 109 \\
91 \rightarrow 95 \\
92 \rightarrow 94\end{array}$ & $\begin{array}{l}1 \mathrm{D} \rightarrow 1 \mathrm{G} \\
2 \mathrm{P} \rightarrow 2 \mathrm{D} \\
2 \mathrm{P} \rightarrow 2 \mathrm{D} \\
2 \mathrm{P} \rightarrow 2 \pi^{*} \\
2 \mathrm{P} \rightarrow 2 \mathrm{D} \\
1 \mathrm{~F} \rightarrow 1 \mathrm{G} \\
1 \mathrm{~F} \rightarrow 2 \mathrm{D} \\
1 \mathrm{~F} \rightarrow 1 \mathrm{G} \\
1 \mathrm{~F} \rightarrow 2 \mathrm{~F} \\
1 \mathrm{~F} \rightarrow 1 \mathrm{G} \\
1 \mathrm{~F} \rightarrow 2 \mathrm{D} \\
1 \mathrm{~F} \rightarrow 1 \mathrm{G} \\
1 \mathrm{~F} \rightarrow 2 \mathrm{D} \\
1 \mathrm{~F} \rightarrow 2 \pi^{*} \\
1 \mathrm{~F} \rightarrow 1 \mathrm{G} \\
1 \mathrm{~F} \rightarrow 1 \mathrm{G}\end{array}$ \\
\hline 222 & 6.9565 & 4.1771 & $\begin{array}{c}-0.10948 \\
0.13244 \\
0.34741 \\
0.11350 \\
0.14833 \\
-0.11593\end{array}$ & $\begin{array}{c}81 \rightarrow 95 \\
83 \rightarrow 102 \\
83 \rightarrow 109 \\
86 \rightarrow 99 \\
86 \rightarrow 109 \\
87 \rightarrow 98\end{array}$ & $\begin{array}{l}1 \mathrm{D} \rightarrow 1 \mathrm{G} \\
2 \mathrm{P} \rightarrow 2 \mathrm{D} \\
2 \mathrm{P} \rightarrow 2 \pi^{*} \\
1 \mathrm{~F} \rightarrow 1 \mathrm{G} \\
1 \mathrm{~F} \rightarrow 2 \pi^{*} \\
1 \mathrm{~F} \rightarrow 1 \mathrm{G}\end{array}$ \\
\hline
\end{tabular}




\begin{tabular}{|c|c|c|c|c|c|}
\hline & & & $\begin{array}{c}-0.13466 \\
-0.12069 \\
-0.13534 \\
-0.13809 \\
-0.11819 \\
-0.11703 \\
0.10009 \\
-0.13411\end{array}$ & $\begin{aligned} 87 \rightarrow 105 \\
88 \rightarrow 101 \\
88 \rightarrow 104 \\
88 \rightarrow 114 \\
89 \rightarrow 100 \\
89 \rightarrow 103 \\
90 \rightarrow 97 \\
91 \rightarrow 96\end{aligned}$ & $\begin{array}{l}1 \mathrm{~F} \rightarrow 2 \mathrm{D} \\
1 \mathrm{~F} \rightarrow 1 \mathrm{G} \\
1 \mathrm{~F} \rightarrow 2 \mathrm{D} \\
1 \mathrm{~F} \rightarrow 2 \mathrm{~F} \\
1 \mathrm{~F} \rightarrow 1 \mathrm{G} \\
1 \mathrm{~F} \rightarrow 2 \mathrm{D} \\
1 \mathrm{~F} \rightarrow 1 \mathrm{G} \\
1 \mathrm{~F} \rightarrow 1 \mathrm{G}\end{array}$ \\
\hline 223 & 6.9624 & 2.3853 & $\begin{array}{c}0.55974 \\
-0.10243 \\
-0.12256\end{array}$ & $\begin{array}{l}84 \rightarrow 109 \\
88 \rightarrow 102 \\
90 \rightarrow 109\end{array}$ & $\begin{array}{c}2 \mathrm{P} \rightarrow 2 \pi^{*} \\
1 \mathrm{~F} \rightarrow 2 \mathrm{D} \\
1 \mathrm{~F} \rightarrow 2 \pi^{*}\end{array}$ \\
\hline 224 & 6.9689 & 1.9399 & $\begin{array}{l}0.56900 \\
0.11488\end{array}$ & $\begin{array}{l}83 \rightarrow 109 \\
86 \rightarrow 109\end{array}$ & $\begin{array}{l}2 \mathrm{P} \rightarrow 2 \pi^{*} \\
1 \mathrm{~F} \rightarrow 2 \pi^{*}\end{array}$ \\
\hline 228 & 7.0138 & 1.3099 & $\begin{array}{c}0.13661 \\
-0.19635 \\
0.37841 \\
0.23795 \\
0.12954 \\
0.14307 \\
-0.12293\end{array}$ & $\begin{array}{c}77 \rightarrow 95 \\
79 \rightarrow 97 \\
83 \rightarrow 113 \\
85 \rightarrow 114 \\
88 \rightarrow 111 \\
88 \rightarrow 115 \\
92 \rightarrow 112\end{array}$ & $\begin{array}{l}1 \mathrm{D} \rightarrow 1 \mathrm{G} \\
1 \mathrm{D} \rightarrow 1 \mathrm{G} \\
2 \mathrm{P} \rightarrow 2 \mathrm{~F} \\
2 \mathrm{P} \rightarrow 2 \mathrm{~F} \\
1 \mathrm{~F} \rightarrow 3 \mathrm{P} \\
1 \mathrm{~F} \rightarrow 3 \mathrm{P} \\
1 \mathrm{~F} \rightarrow 3 \mathrm{P}\end{array}$ \\
\hline 230 & 7.0275 & 4.3596 & $\begin{array}{c}0.11735 \\
0.18668 \\
-0.15895 \\
0.10796 \\
-0.12519 \\
0.12021 \\
-0.13231 \\
-0.13242 \\
-0.11591 \\
0.12633 \\
0.11222 \\
0.10072 \\
-0.11439 \\
0.11691 \\
-0.10293 \\
-0.13012 \\
-0.10303\end{array}$ & $\begin{array}{c}78 \rightarrow 94 \\
79 \rightarrow 97 \\
81 \rightarrow 93 \\
85 \rightarrow 107 \\
85 \rightarrow 114 \\
86 \rightarrow 100 \\
86 \rightarrow 103 \\
86 \rightarrow 113 \\
87 \rightarrow 101 \\
87 \rightarrow 106 \\
88 \rightarrow 98 \\
88 \rightarrow 108 \\
88 \rightarrow 115 \\
89 \rightarrow 99 \\
89 \rightarrow 102 \\
90 \rightarrow 93 \\
92 \rightarrow 97\end{array}$ & $\begin{array}{l}1 \mathrm{D} \rightarrow 1 \mathrm{G} \\
1 \mathrm{D} \rightarrow 1 \mathrm{G} \\
1 \mathrm{D} \rightarrow 1 \mathrm{G} \\
2 \mathrm{P} \rightarrow 3 \mathrm{~S} \\
2 \mathrm{P} \rightarrow 2 \mathrm{~F} \\
1 \mathrm{~F} \rightarrow 1 \mathrm{G} \\
1 \mathrm{~F} \rightarrow 2 \mathrm{D} \\
1 \mathrm{~F} \rightarrow 2 \mathrm{~F} \\
1 \mathrm{~F} \rightarrow 1 \mathrm{G} \\
1 \mathrm{~F} \rightarrow 2 \mathrm{D} \\
1 \mathrm{~F} \rightarrow 1 \mathrm{G} \\
1 \mathrm{~F} \rightarrow 2 \pi^{*} \\
1 \mathrm{~F} \rightarrow 2 \mathrm{~F} \\
1 \mathrm{~F} \rightarrow 1 \mathrm{G} \\
1 \mathrm{~F} \rightarrow 2 \mathrm{D} \\
1 \mathrm{~F} \rightarrow 1 \mathrm{G} \\
1 \mathrm{~F} \rightarrow 1 \mathrm{G}\end{array}$ \\
\hline
\end{tabular}




\begin{tabular}{|c|c|c|c|c|c|c|c|c|}
\hline \multirow[t]{2}{*}{$\begin{array}{c}\text { Excited } \\
\text { States }\end{array}$} & \multicolumn{3}{|c|}{$\begin{array}{c}\text { Transition electric dipole } \\
\text { moment }(\mathrm{au})\end{array}$} & \multirow[t]{2}{*}{$\begin{array}{c}\text { Energy } \\
(\mathrm{eV})\end{array}$} & \multirow[t]{2}{*}{$\begin{array}{l}\text { Oscillator } \\
\text { Strength }\end{array}$} & \multirow[t]{2}{*}{ Weight } & \multirow[t]{2}{*}{ Transitions } & \multirow[t]{2}{*}{ Orbitals } \\
\hline & $\mathrm{X}$ & $\mathrm{Y}$ & $\mathrm{Z}$ & & & & & \\
\hline 153 & -0.0009 & 0.2775 & -0.0001 & 5.9177 & 0.0112 & $\begin{array}{c}0.18933 \\
-0.31868 \\
0.13338 \\
-0.14818 \\
0.37852 \\
0.19265 \\
0.15114 \\
0.25256\end{array}$ & $\begin{array}{c}77 \rightarrow 93 \\
82 \rightarrow 93 \\
84 \rightarrow 112 \\
85 \rightarrow 108 \\
87 \rightarrow 108 \\
87 \rightarrow 111 \\
87 \rightarrow 115 \\
92 \rightarrow 108\end{array}$ & $\begin{array}{c}1 \mathrm{D} \rightarrow 1 \mathrm{G} \\
2 \mathrm{~S} \rightarrow 1 \mathrm{G} \\
2 \mathrm{P} \rightarrow 3 \mathrm{P} \\
2 \mathrm{P} \rightarrow 2 \pi^{*} \\
1 \mathrm{~F} \rightarrow 2 \pi^{*} \\
1 \mathrm{~F} \rightarrow 3 \mathrm{P} \\
1 \mathrm{~F} \rightarrow 2 \mathrm{~F} \\
1 \mathrm{~F} \rightarrow 2 \pi^{*}\end{array}$ \\
\hline 154 & -0.5634 & -0.0005 & -0.0000 & 5.9190 & 0.0460 & $\begin{array}{c}-0.40961 \\
0.42582 \\
0.21123 \\
\end{array}$ & $\begin{array}{c}82 \rightarrow 97 \\
90 \rightarrow 108 \\
90 \rightarrow 111\end{array}$ & $\begin{array}{c}2 \mathrm{~S} \rightarrow 1 \mathrm{G} \\
1 \mathrm{~F} \rightarrow 2 \pi^{*} \\
1 \mathrm{~F} \rightarrow 3 \mathrm{P}\end{array}$ \\
\hline 165 & -0.5148 & -0.0000 & -0.0000 & 6.1502 & 0.0399 & $\begin{array}{c}0.16778 \\
0.40635 \\
0.15696 \\
0.24619 \\
0.24223 \\
-0.22195 \\
0.17228 \\
\end{array}$ & $\begin{array}{c}82 \rightarrow 97 \\
86 \rightarrow 110 \\
88 \rightarrow 108 \\
88 \rightarrow 111 \\
90 \rightarrow 108 \\
90 \rightarrow 111 \\
92 \rightarrow 112\end{array}$ & $\begin{array}{c}2 \mathrm{~S} \rightarrow 1 \mathrm{G} \\
1 \mathrm{~F} \rightarrow 3 \mathrm{P} \\
1 \mathrm{~F} \rightarrow 2 \pi^{*} \\
1 \mathrm{~F} \rightarrow 3 \mathrm{P} \\
1 \mathrm{~F} \rightarrow 2 \pi^{*} \\
1 \mathrm{~F} \rightarrow 3 \mathrm{P} \\
1 \mathrm{~F} \rightarrow 3 \mathrm{P}\end{array}$ \\
\hline 169 & 0.7957 & -0.0000 & -0.0000 & 6.2042 & 0.0962 & $\begin{array}{c}-0.10890 \\
-0.10650 \\
0.12224 \\
-0.21662 \\
0.13517 \\
0.36077 \\
-0.27973 \\
0.18560 \\
-0.28467 \\
\end{array}$ & $\begin{array}{c}82 \rightarrow 95 \\
84 \rightarrow 108 \\
86 \rightarrow 110 \\
87 \rightarrow 112 \\
88 \rightarrow 108 \\
88 \rightarrow 111 \\
90 \rightarrow 108 \\
90 \rightarrow 111 \\
92 \rightarrow 112\end{array}$ & $\begin{array}{c}2 \mathrm{~S} \rightarrow 1 \mathrm{G} \\
2 \mathrm{P} \rightarrow 2 \pi^{*} \\
1 \mathrm{~F} \rightarrow 3 \mathrm{P} \\
1 \mathrm{~F} \rightarrow 3 \mathrm{P} \\
1 \mathrm{~F} \rightarrow 2 \pi^{*} \\
1 \mathrm{~F} \rightarrow 3 \mathrm{P} \\
1 \mathrm{~F} \rightarrow 2 \pi^{*} \\
1 \mathrm{~F} \rightarrow 3 \mathrm{P} \\
1 \mathrm{~F} \rightarrow 3 \mathrm{P}\end{array}$ \\
\hline 175 & -0.0000 & -0.4919 & 0.0003 & 6.3686 & 0.0377 & $\begin{array}{l}0.14938 \\
0.18436 \\
0.43086 \\
0.18924 \\
0.14886 \\
0.34927 \\
\end{array}$ & $\begin{array}{c}77 \rightarrow 93 \\
82 \rightarrow 93 \\
85 \rightarrow 108 \\
87 \rightarrow 115 \\
92 \rightarrow 108 \\
92 \rightarrow 111\end{array}$ & $\begin{array}{c}1 \mathrm{D} \rightarrow 1 \mathrm{G} \\
2 \mathrm{~S} \rightarrow 1 \mathrm{G} \\
2 \mathrm{P} \rightarrow 2 \pi^{*} \\
1 \mathrm{~F} \rightarrow 2 \mathrm{~F} \\
1 \mathrm{~F} \rightarrow 2 \pi^{*} \\
1 \mathrm{~F} \rightarrow 3 \mathrm{P}\end{array}$ \\
\hline
\end{tabular}




\begin{tabular}{|c|c|c|c|c|c|}
\hline $\begin{array}{c}\text { Excited } \\
\text { States }\end{array}$ & $\begin{array}{c}\text { Energy } \\
(\mathrm{eV})\end{array}$ & $\begin{array}{l}\text { Oscillator } \\
\text { Strength }\end{array}$ & Weight & Transitions & Orbitals \\
\hline 203 & 6.9547 & 8.2360 & $\begin{array}{c}0.11475 \\
-0.13265 \\
-0.13324 \\
0.10922 \\
-0.15428 \\
0.16565 \\
-0.10405 \\
0.16569 \\
-0.10057 \\
-0.16601 \\
0.20463 \\
-0.16604 \\
0.20424 \\
-0.11316 \\
-0.11867 \\
0.11349 \\
-0.11998 \\
-0.19316 \\
-0.11818 \\
0.13818\end{array}$ & $\begin{array}{c}75 \rightarrow 103 \\
76 \rightarrow 98 \\
77 \rightarrow 99 \\
78 \rightarrow 86 \\
78 \rightarrow 97 \\
79 \rightarrow 94 \\
79 \rightarrow 96 \\
80 \rightarrow 93 \\
80 \rightarrow 95 \\
81 \rightarrow 91 \\
81 \rightarrow 99 \\
82 \rightarrow 92 \\
82 \rightarrow 98 \\
83 \rightarrow 88 \\
83 \rightarrow 93 \\
84 \rightarrow 87 \\
84 \rightarrow 94 \\
85 \rightarrow 86 \\
85 \rightarrow 97 \\
85 \rightarrow 121\end{array}$ & $\begin{array}{l}2 \mathrm{P} \rightarrow 3 \mathrm{P} \\
2 \mathrm{P} \rightarrow 1 \mathrm{G} \\
2 \mathrm{P} \rightarrow 1 \mathrm{G} \\
2 \mathrm{P} \rightarrow 2 \mathrm{D} \\
2 \mathrm{P} \rightarrow 1 \mathrm{G} \\
1 \mathrm{~F} \rightarrow 1 \mathrm{G} \\
1 \mathrm{~F} \rightarrow 1 \mathrm{G} \\
1 \mathrm{~F} \rightarrow 1 \mathrm{G} \\
1 \mathrm{~F} \rightarrow 1 \mathrm{G} \\
1 \mathrm{~F} \rightarrow 1 \mathrm{G} \\
1 \mathrm{~F} \rightarrow 1 \mathrm{G} \\
1 \mathrm{~F} \rightarrow 1 \mathrm{G} \\
1 \mathrm{~F} \rightarrow 1 \mathrm{G} \\
1 \mathrm{~F} \rightarrow 2 \mathrm{D} \\
1 \mathrm{~F} \rightarrow 1 \mathrm{G} \\
1 \mathrm{~F} \rightarrow 2 \mathrm{D} \\
1 \mathrm{~F} \rightarrow 1 \mathrm{G} \\
1 \mathrm{~F} \rightarrow 2 \mathrm{D} \\
1 \mathrm{~F} \rightarrow 1 \mathrm{G} \\
1 \mathrm{~F} \rightarrow 3 \mathrm{D}\end{array}$ \\
\hline 204 & 6.9550 & 8.2369 & $\begin{array}{c}0.11468 \\
0.13437 \\
0.13500 \\
-0.13415 \\
0.16659 \\
0.15819 \\
0.16659 \\
0.15463 \\
0.16627 \\
0.17562 \\
0.16627 \\
0.11123 \\
0.11680 \\
-0.10745 \\
0.11820 \\
0.11682 \\
-0.11762\end{array}$ & $\begin{array}{l}75 \rightarrow 102 \\
76 \rightarrow 96 \\
77 \rightarrow 95 \\
78 \rightarrow 99 \\
79 \rightarrow 92 \\
79 \rightarrow 95 \\
80 \rightarrow 91 \\
80 \rightarrow 96 \\
81 \rightarrow 93 \\
81 \rightarrow 97 \\
82 \rightarrow 94 \\
83 \rightarrow 87 \\
83 \rightarrow 89 \\
84 \rightarrow 88 \\
84 \rightarrow 90 \\
85 \rightarrow 89 \\
85 \rightarrow 91\end{array}$ & $\begin{array}{l}2 \mathrm{~S} \rightarrow 3 \mathrm{P} \\
2 \mathrm{P} \rightarrow 1 \mathrm{G} \\
2 \mathrm{P} \rightarrow 1 \mathrm{G} \\
2 \mathrm{P} \rightarrow 1 \mathrm{G} \\
1 \mathrm{~F} \rightarrow 1 \mathrm{G} \\
1 \mathrm{~F} \rightarrow 1 \mathrm{G} \\
1 \mathrm{~F} \rightarrow 1 \mathrm{G} \\
1 \mathrm{~F} \rightarrow 1 \mathrm{G} \\
1 \mathrm{~F} \rightarrow 1 \mathrm{G} \\
1 \mathrm{~F} \rightarrow 1 \mathrm{G} \\
1 \mathrm{~F} \rightarrow 1 \mathrm{G} \\
1 \mathrm{~F} \rightarrow 2 \mathrm{D} \\
1 \mathrm{~F} \rightarrow 2 \mathrm{D} \\
1 \mathrm{~F} \rightarrow 2 \mathrm{D} \\
1 \mathrm{~F} \rightarrow 2 \mathrm{D} \\
1 \mathrm{~F} \rightarrow 2 \mathrm{D} \\
1 \mathrm{~F} \rightarrow 1 \mathrm{G}\end{array}$ \\
\hline 205 & 6.9550 & 8.2369 & $\begin{array}{c}0.11468 \\
0.10850 \\
-0.13308 \\
0.13445\end{array}$ & $\begin{array}{c}75 \rightarrow 101 \\
76 \rightarrow 88 \\
76 \rightarrow 95 \\
77 \rightarrow 96\end{array}$ & $\begin{array}{l}2 \mathrm{~S} \rightarrow 3 \mathrm{P} \\
2 \mathrm{P} \rightarrow 2 \mathrm{D} \\
2 \mathrm{P} \rightarrow 1 \mathrm{G} \\
2 \mathrm{P} \rightarrow 1 \mathrm{G}\end{array}$ \\
\hline
\end{tabular}




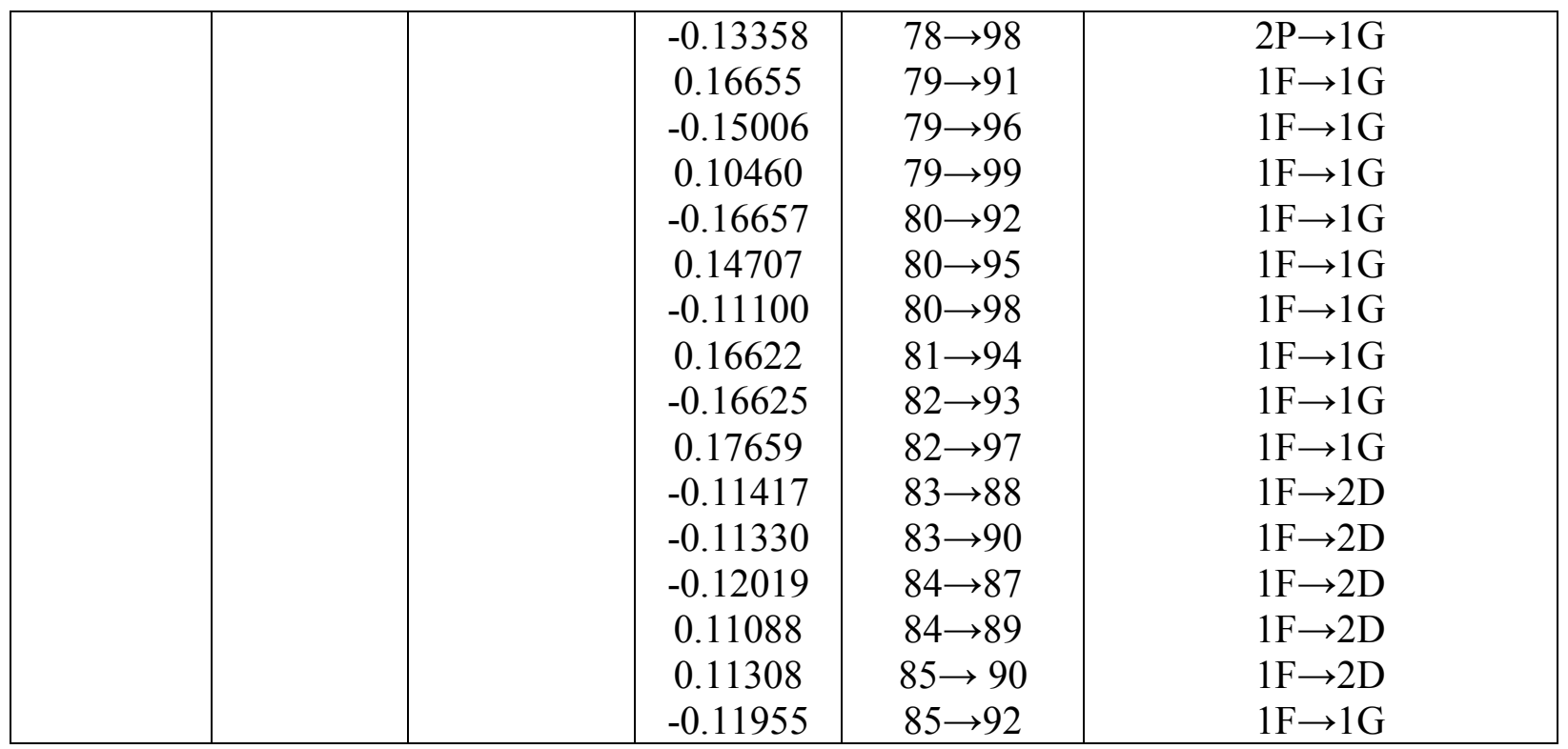
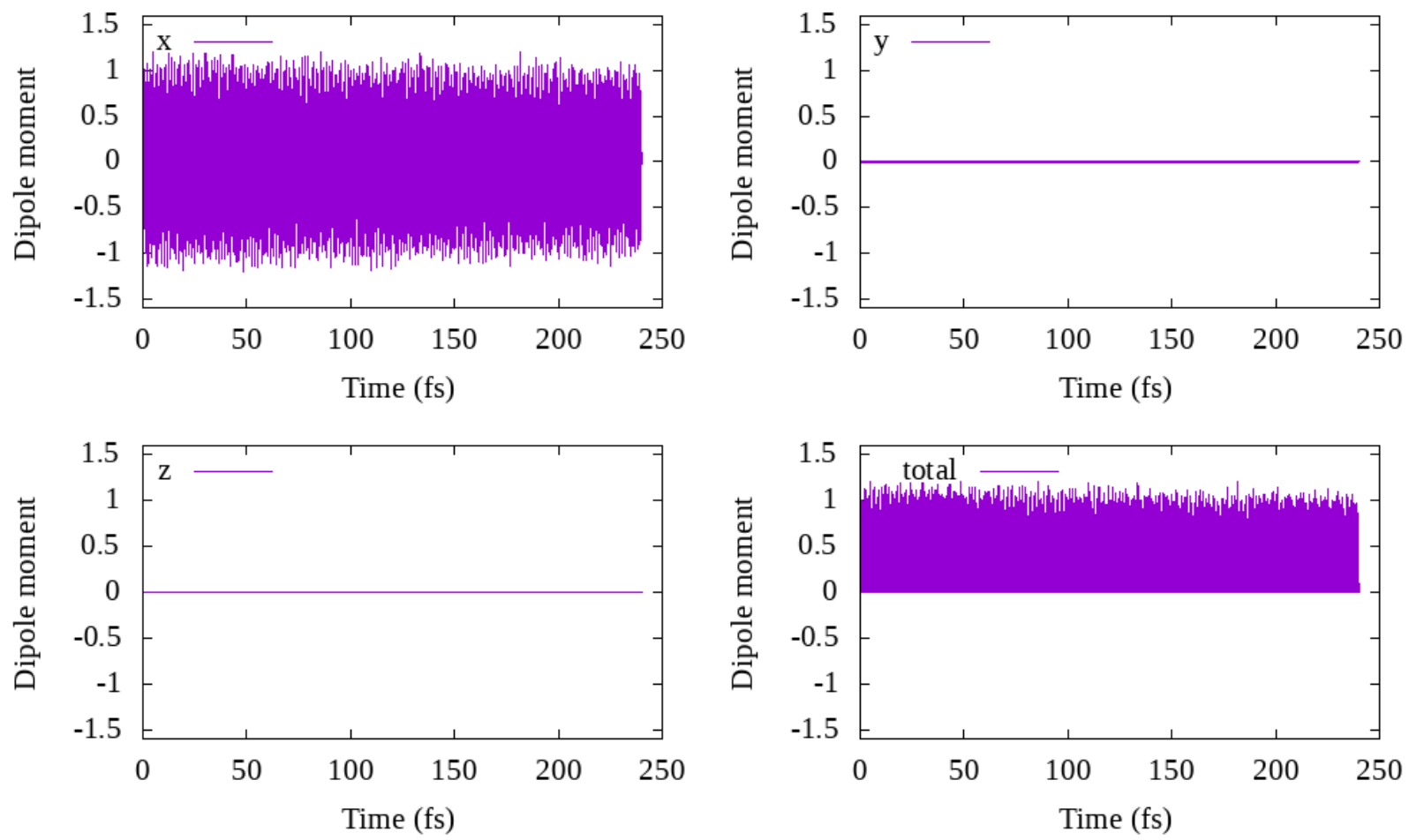

Figure S1. Dipole moment (Debye) induced in the $\mathrm{Al}_{13}{ }^{-1}$ system on applying a 0.001 au step field along the $\mathrm{x}$-direction. 

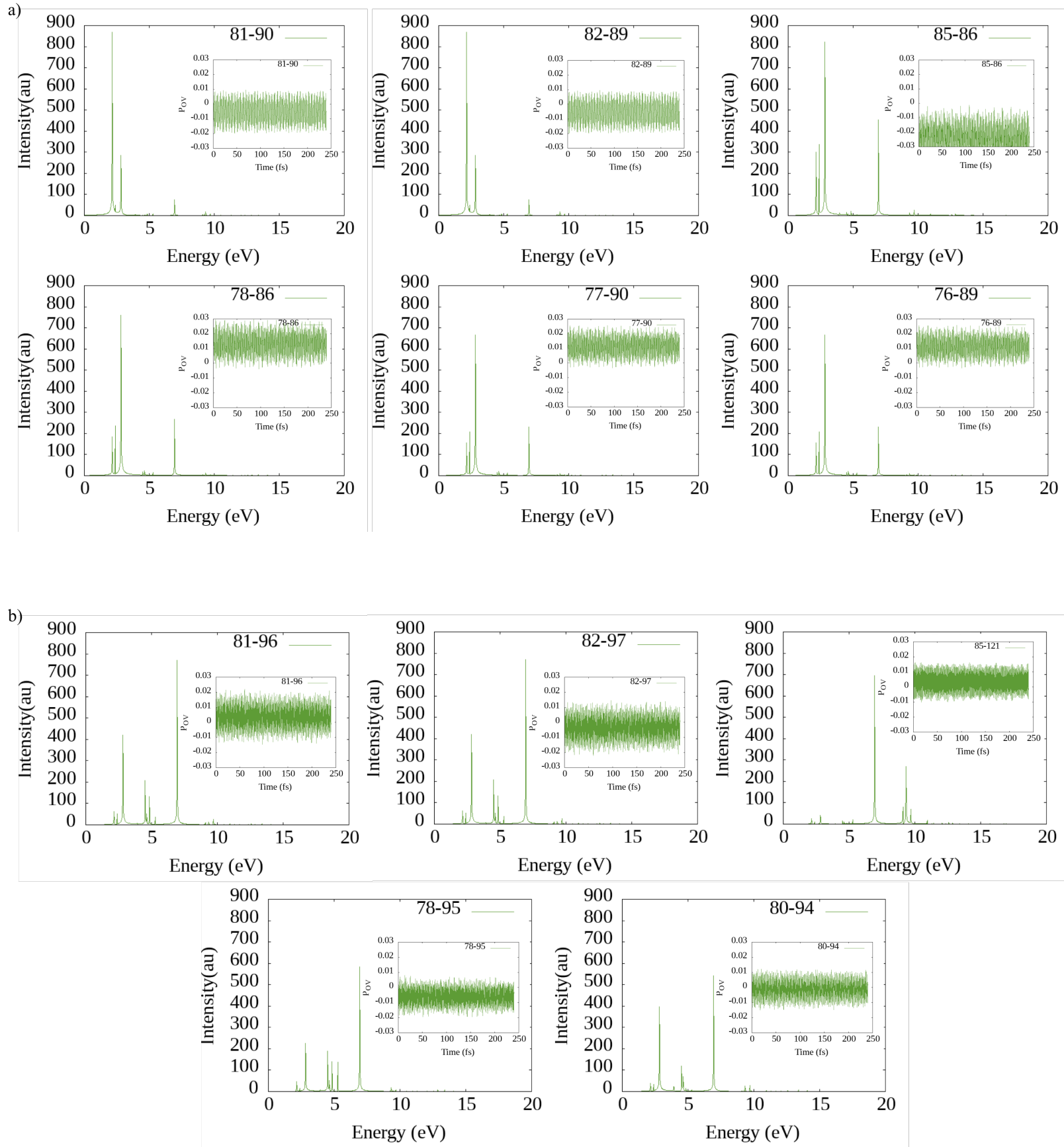

Figure S2. (a) FT peaks and their corresponding POV elements for the transitions that correspond to the peaks at $2-2.5 \mathrm{eV}$. (b) FT peaks and their corresponding Pov for the transitions that corresponds to the peak at $7 \mathrm{eV}$. These are obtained from the application of a step electric field of 0.001 au along the $\mathrm{z}$-direction in the $\mathrm{Al}_{13}{ }^{-1}$ system. 

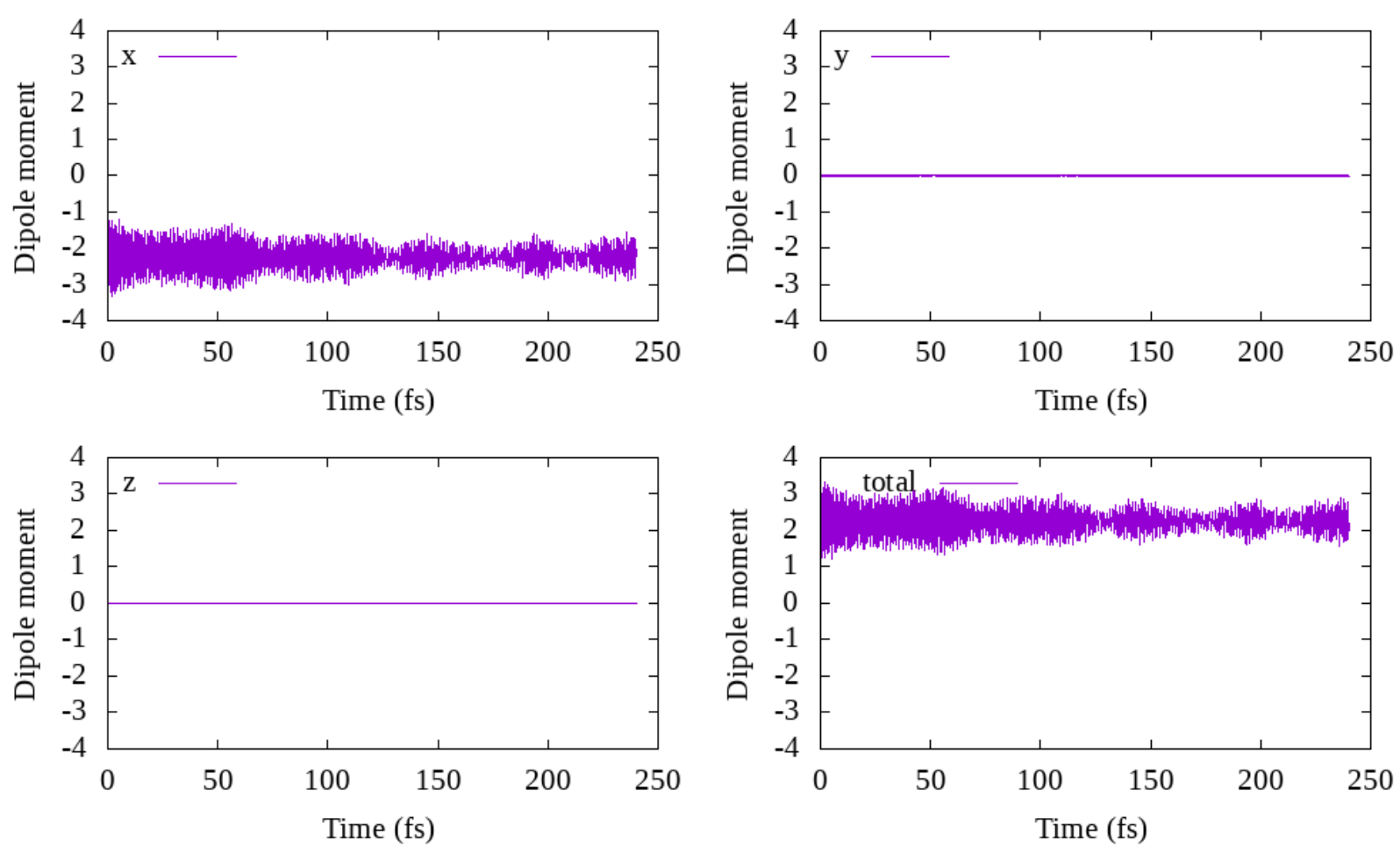

Figure S3. Dipole moment (Debye) induced in $\left[\mathrm{Al}_{13} \mathrm{~N}_{2}\right]^{-1}$ on applying a 0.001 au step field along the $\mathrm{x}$-direction.
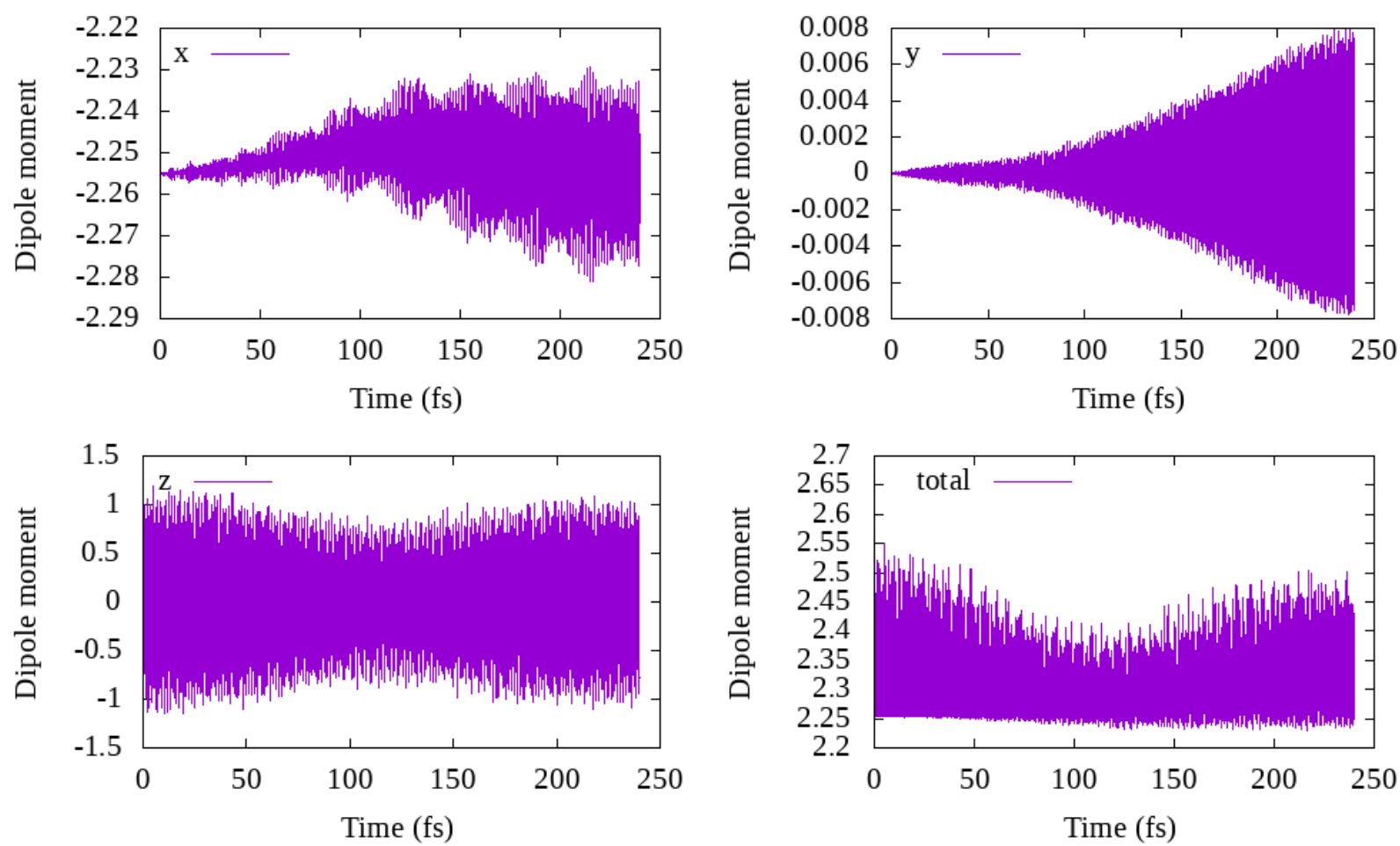

Figure S4. Dipole moment (Debye) induced from the application of 0.001 au step laser field along the $\mathrm{z}$-direction in $\left[\mathrm{Al}_{13} \mathrm{~N}_{2}\right]^{-1}$. 

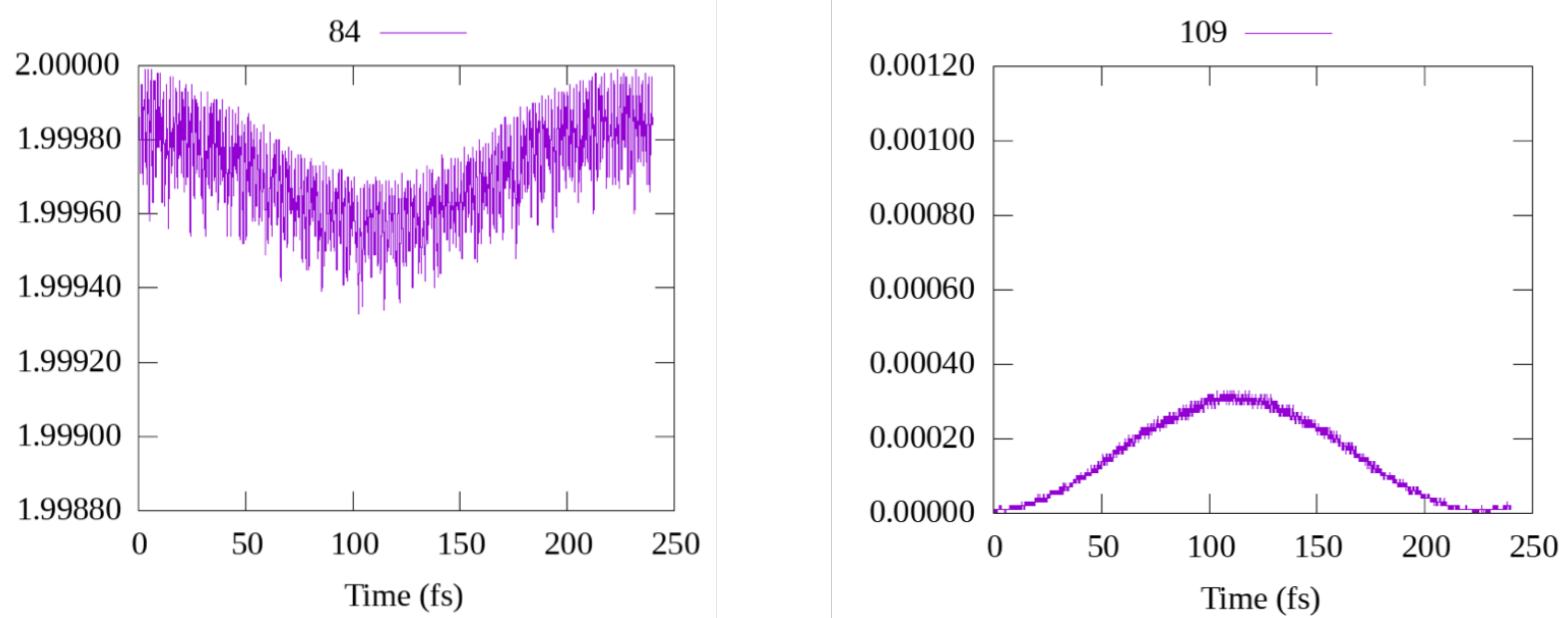

Figure S5. Orbital occupation numbers in orbital 84 and orbital 109 after the application of 0.001 au step field along the z-direction in $\left[\mathrm{Al}_{13} \mathrm{~N}_{2}\right]^{-1}$. 

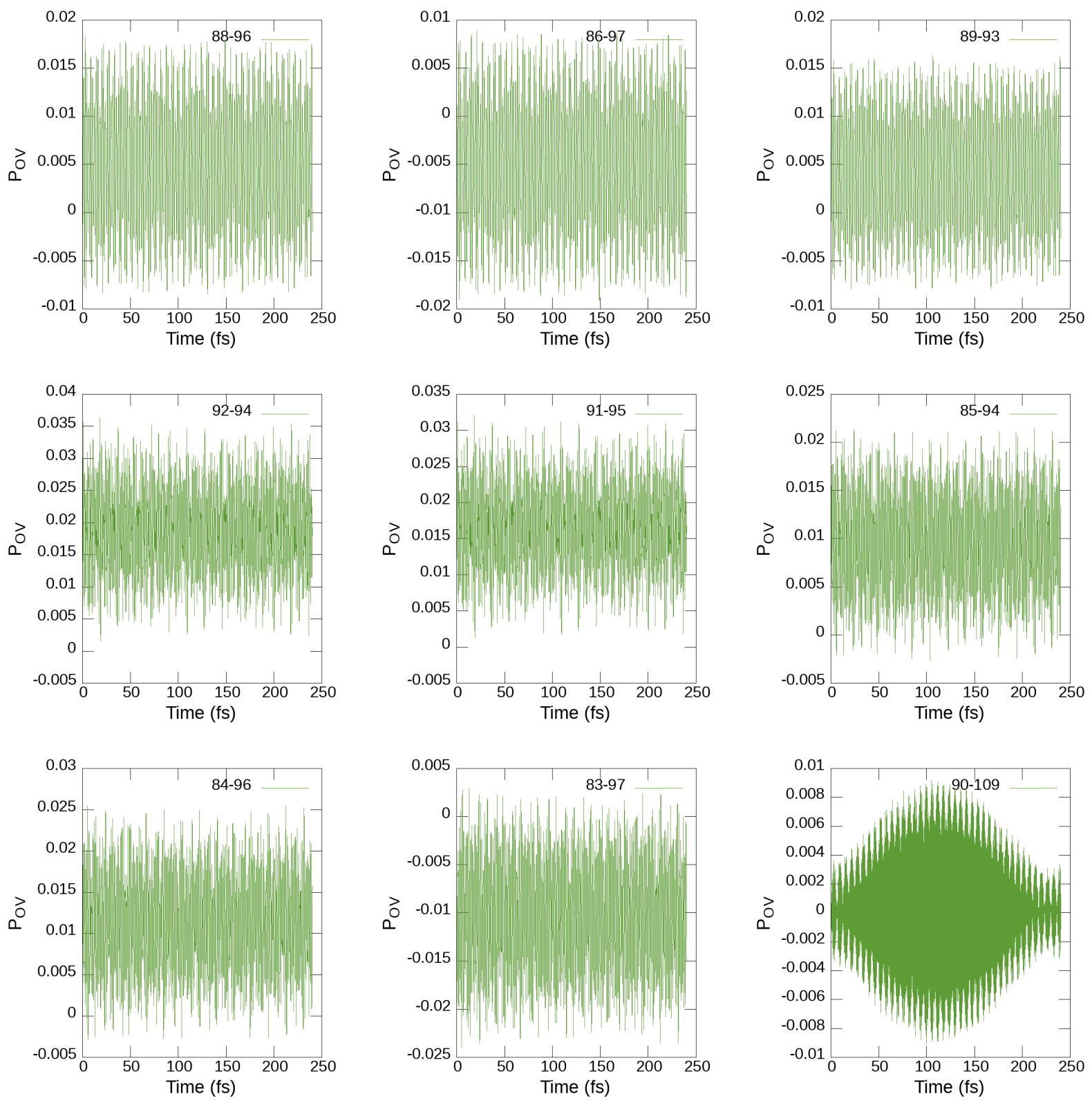

a) 

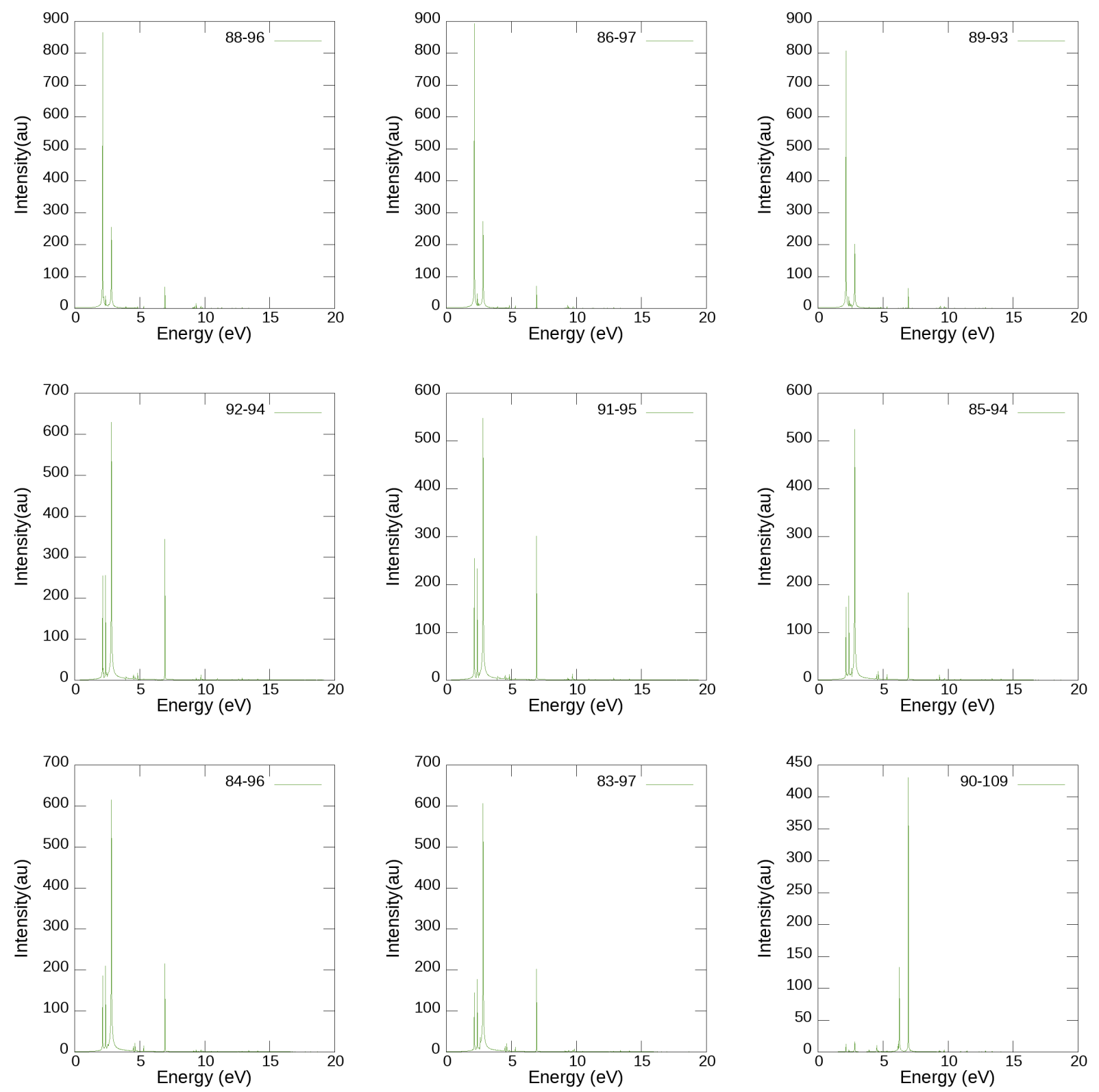

b) 

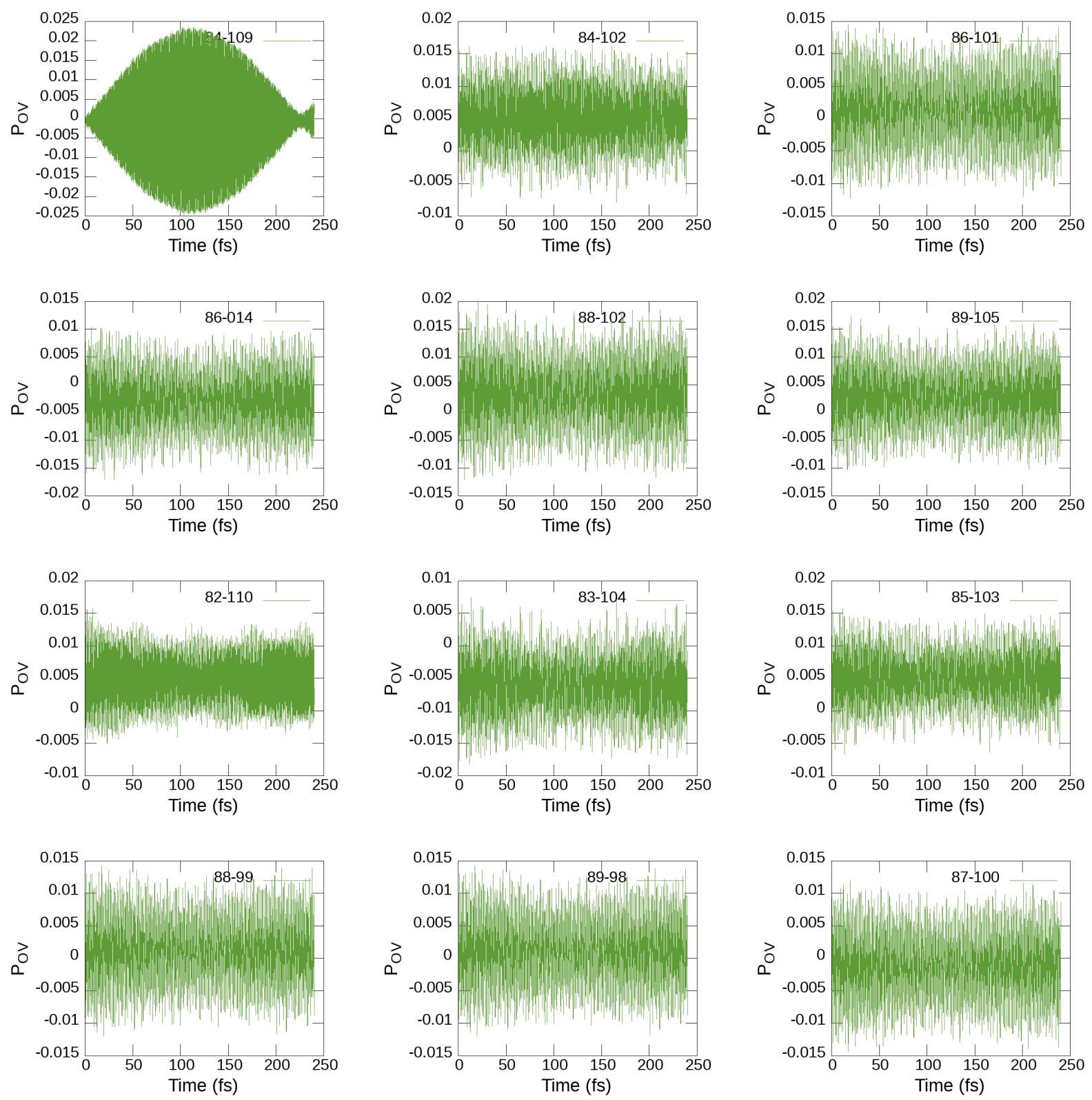

c) 

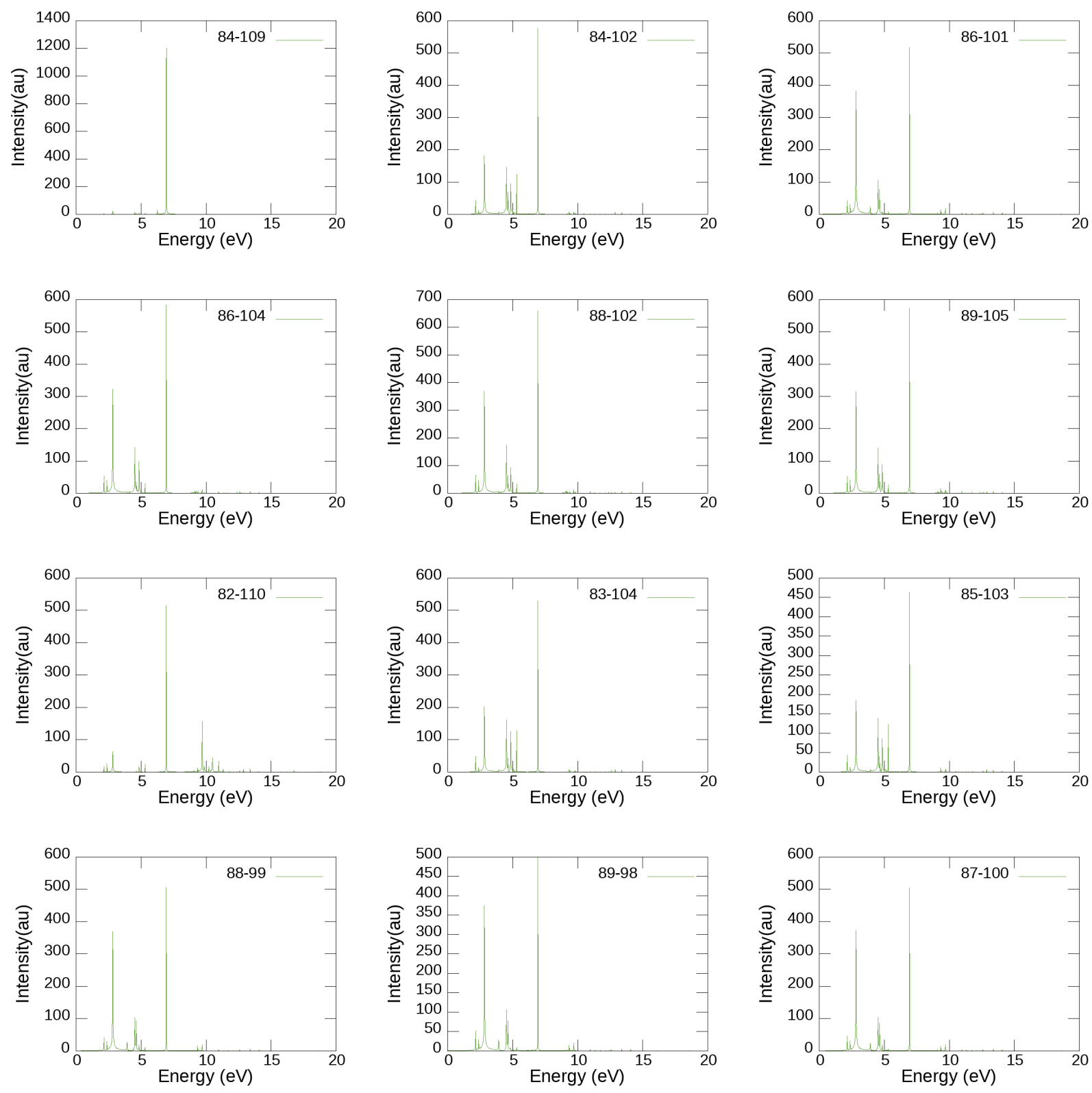

d)

Figure S6. P $_{\text {Ov }}$ for different transitions that contribute to the major peaks at $2-2.5 \mathrm{eV}$ and $6 \mathrm{eV}$ (a), and the FT peaks for the corresponding transitions (b). Only the $90 \rightarrow 109$ transition contributes to the FT peak at $6 \mathrm{eV}$. Pov $_{\mathrm{OV}}$ for different transitions that contribute to the major peaks at $7 \mathrm{eV}$ (c) and the FT peaks for the corresponding transitions (d). These are obtained from the application of a 0.001 au step field along the $\mathrm{z}$-direction in the $\left[\mathrm{Al}_{13} \mathrm{~N}_{2}\right]^{-1}$ system. 

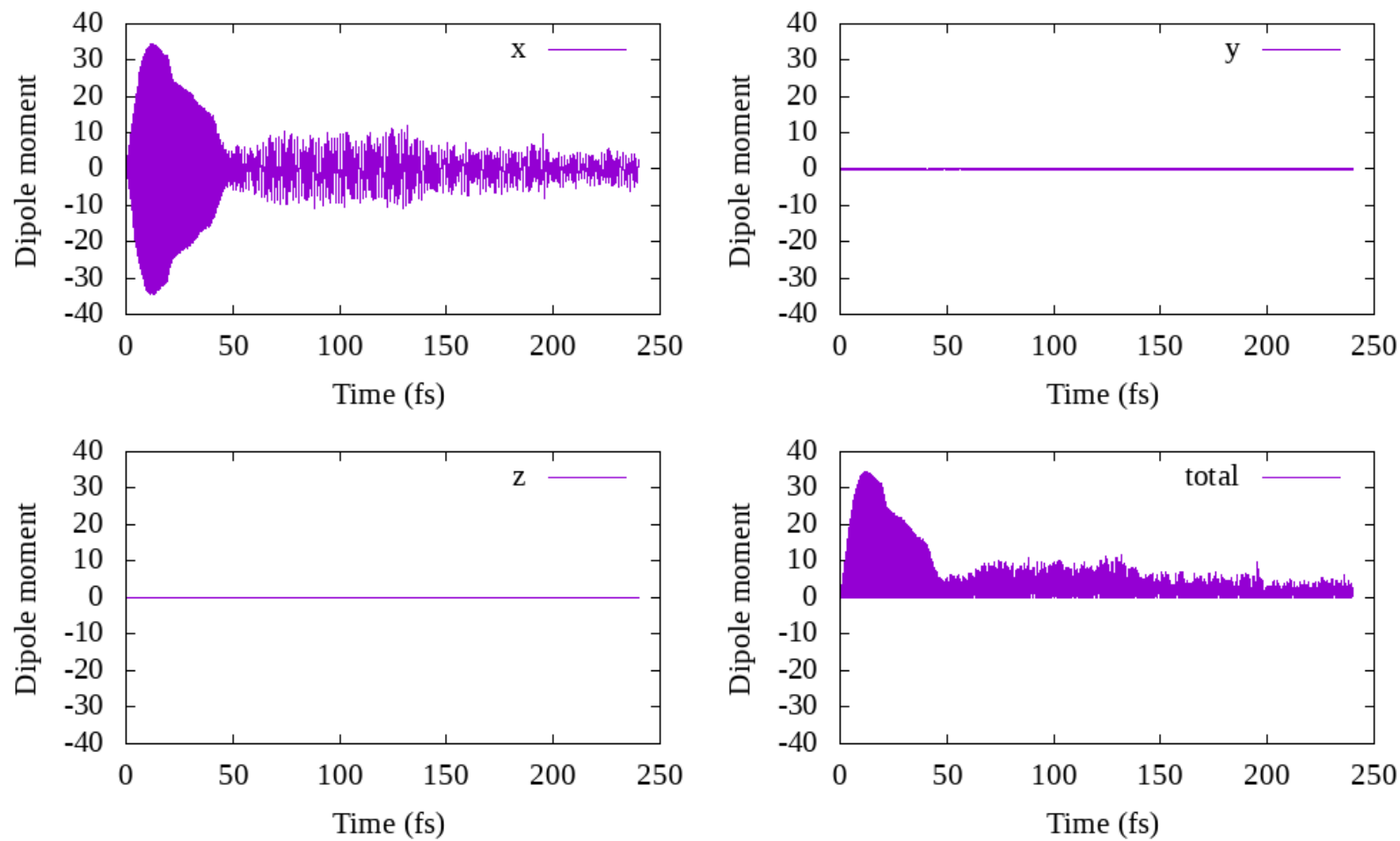

Figure S7. Variation of different components of the dipole moment (Debye) with time after the application of a 0.001 au trapezoid field with an energy of $7 \mathrm{eV}$ along the $\mathrm{x}$-direction to the $\mathrm{Al}_{13^{-1}}$ system. 

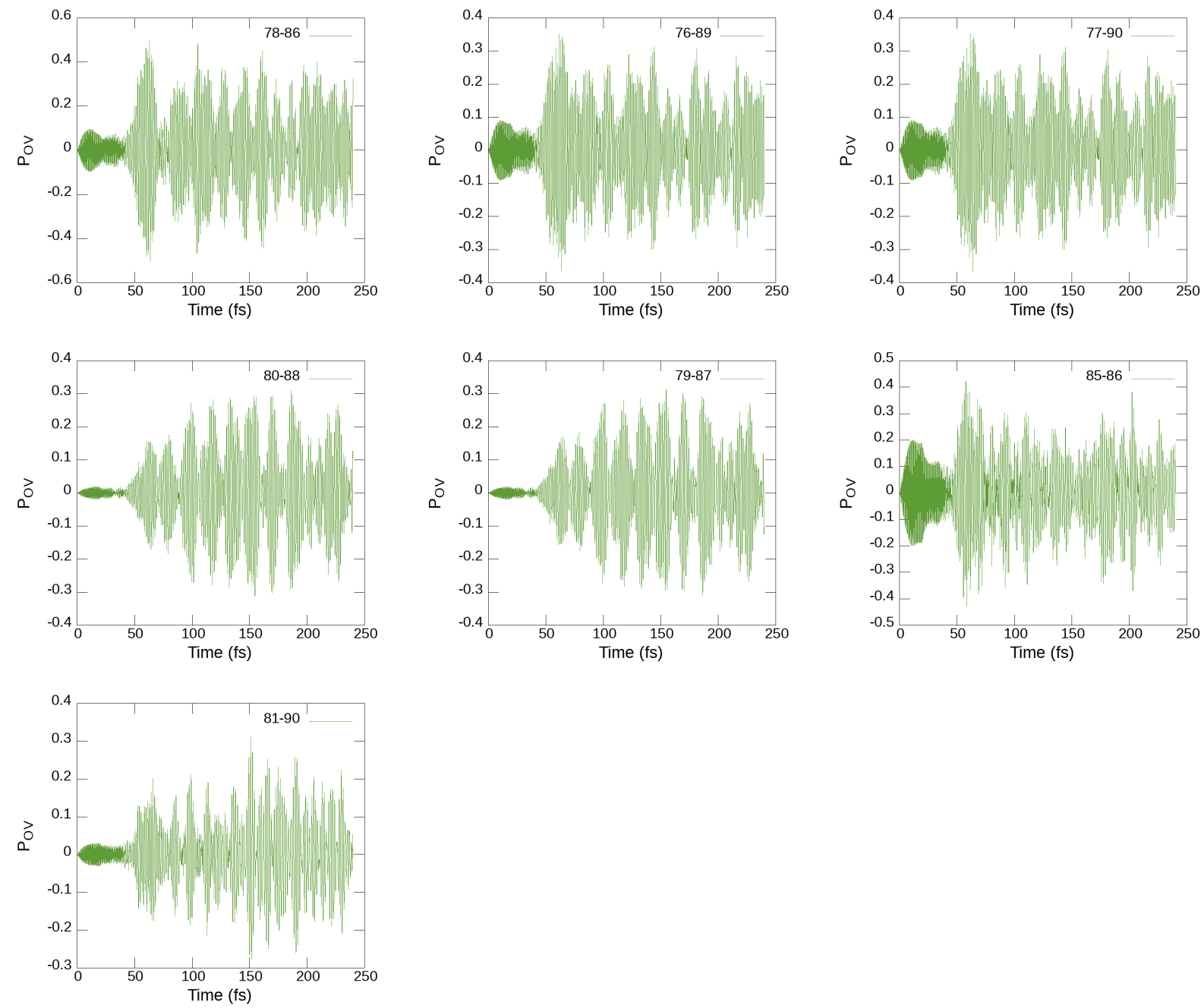

a) 

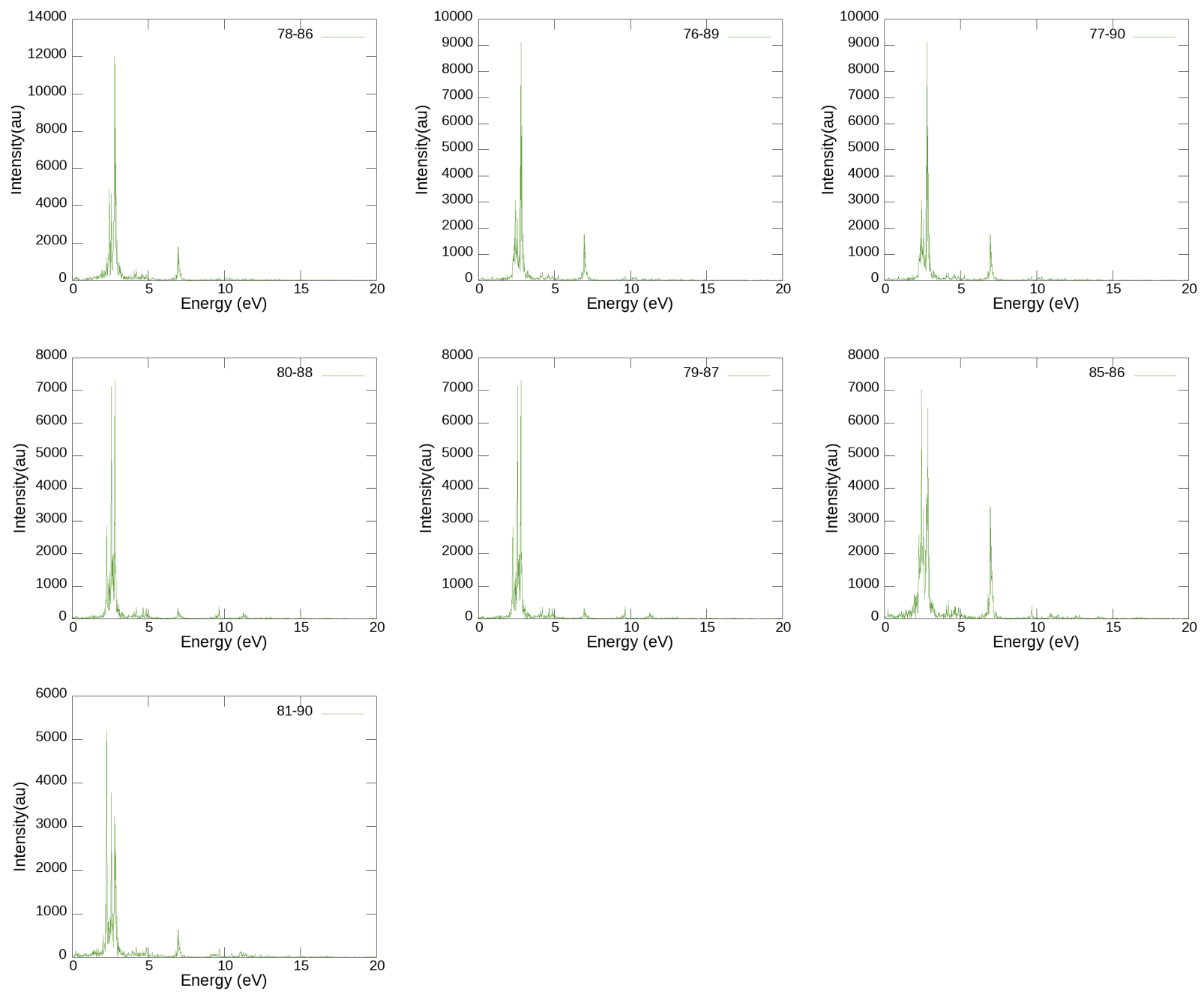

b) 

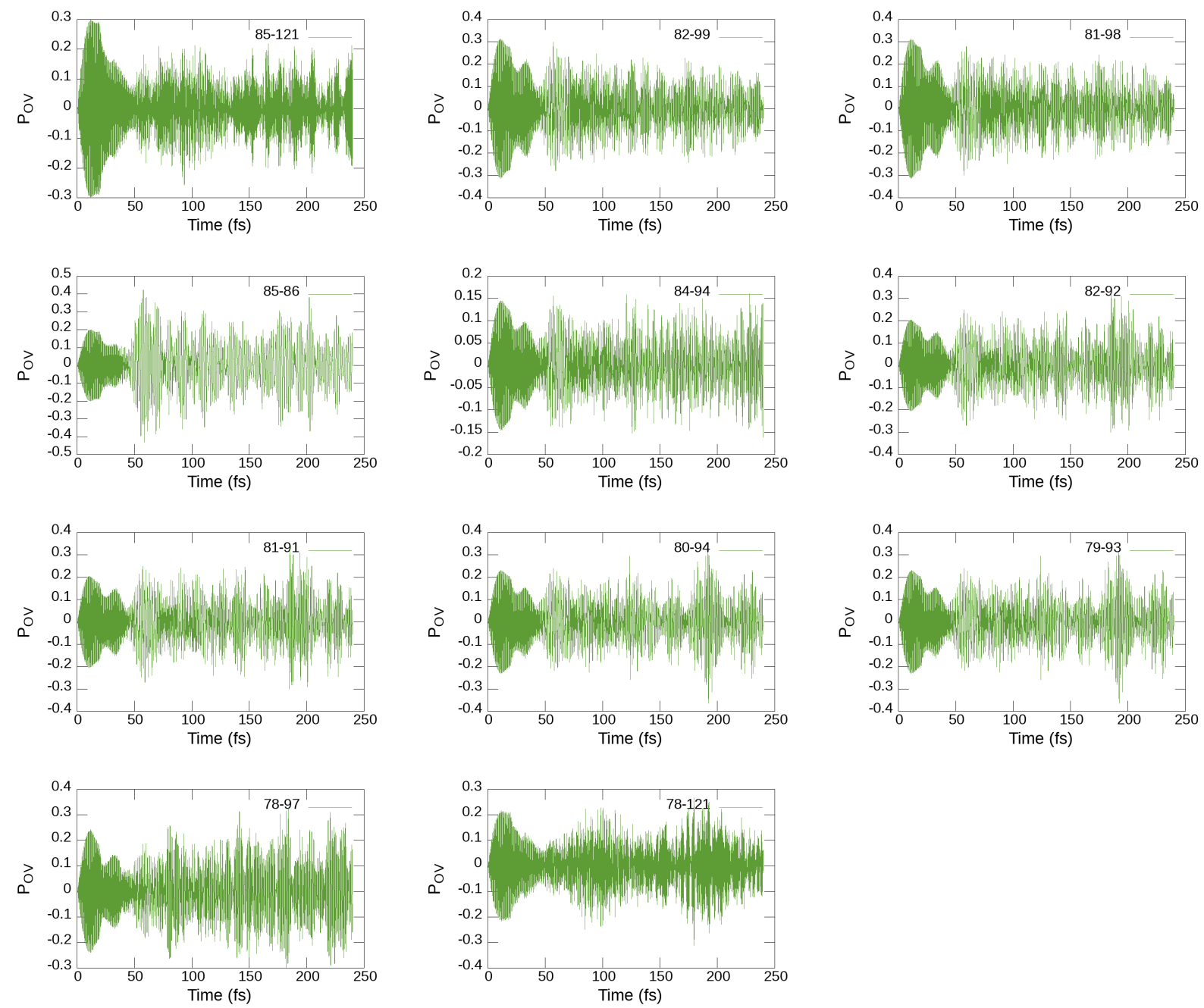

c) 

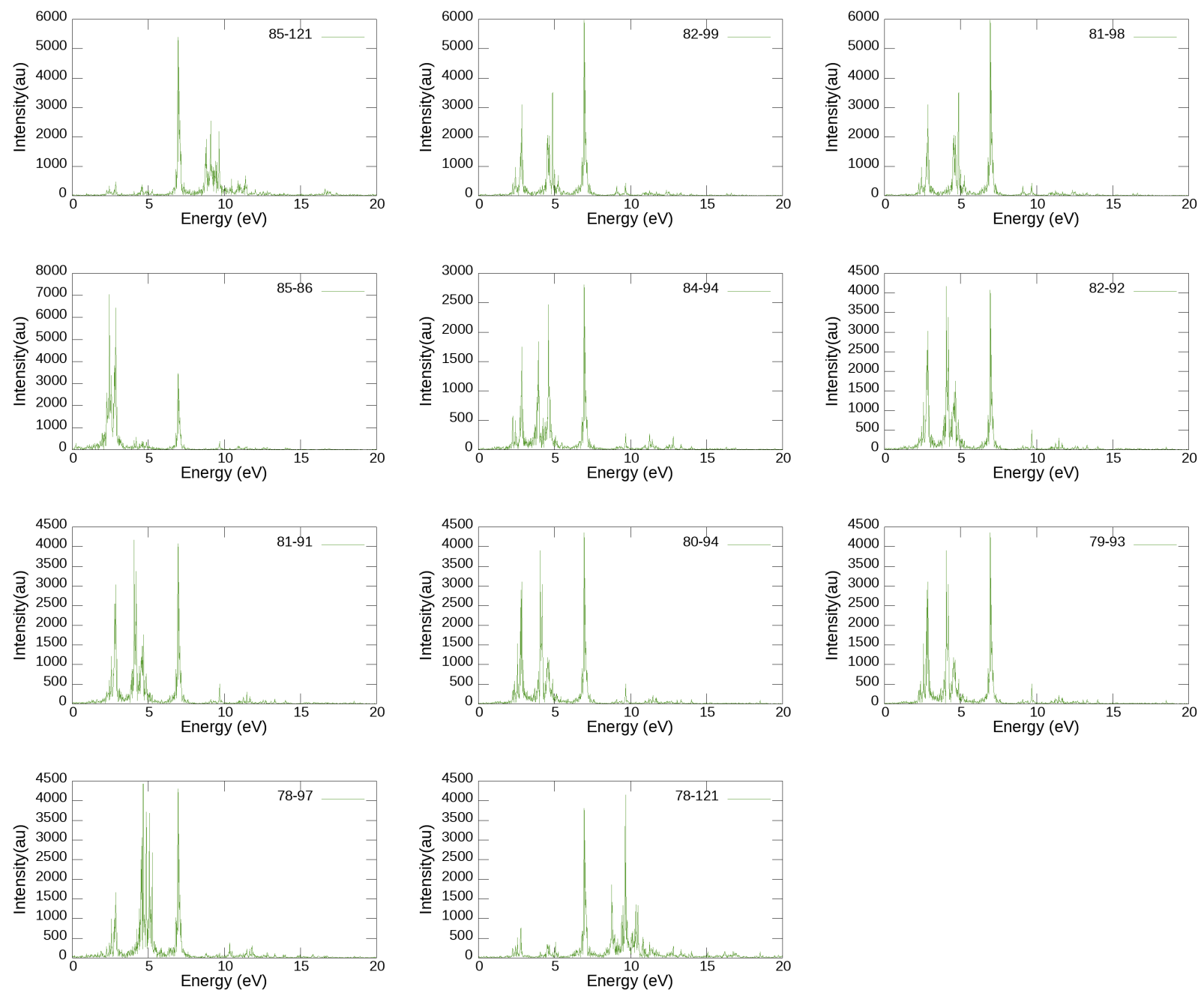

d)

Figure S8. $P_{\text {Ov }}$ for different transitions that contribute the major peaks at 2-3 eV (a), and the FT peaks for the corresponding transitions (b). Pov for different transitions that contribute the major peaks at $7 \mathrm{eV}$ (c) and the FT peaks for the corresponding transitions (d). These are obtained from the application of a 0.001 au trapezoid laser field of frequency corresponding to $7 \mathrm{eV}$ along the $\mathrm{z}-$ direction in the $\left[\mathrm{Al}_{13}\right]^{-1}$ system.

Table S7. Dynamic and static polarizabilities of the $\left[\mathrm{Al}_{13} \mathrm{~N}_{2}\right]^{-1}$ with $\mathrm{C}_{2 \mathrm{v}}$ symmetry. Frequency corresponding to $0 \mathrm{eV}$ (static polarizability):

$\begin{array}{lll}558.54 & 0.0000 & 0.0000 \\ 0.0000 & 560.89 & 0.0000 \\ 0.0000 & 0.0000 & 571.05\end{array}$

Frequency corresponding to $7 \mathrm{eV}$ or 0.257 au (dynamic polarizability):

$\begin{array}{rrr}-31503.7 & 0.00000 & 0.00000 \\ 0.00000 & -11894.6 & 0.00000 \\ 0.00000 & 0.00000 & 11444.5\end{array}$



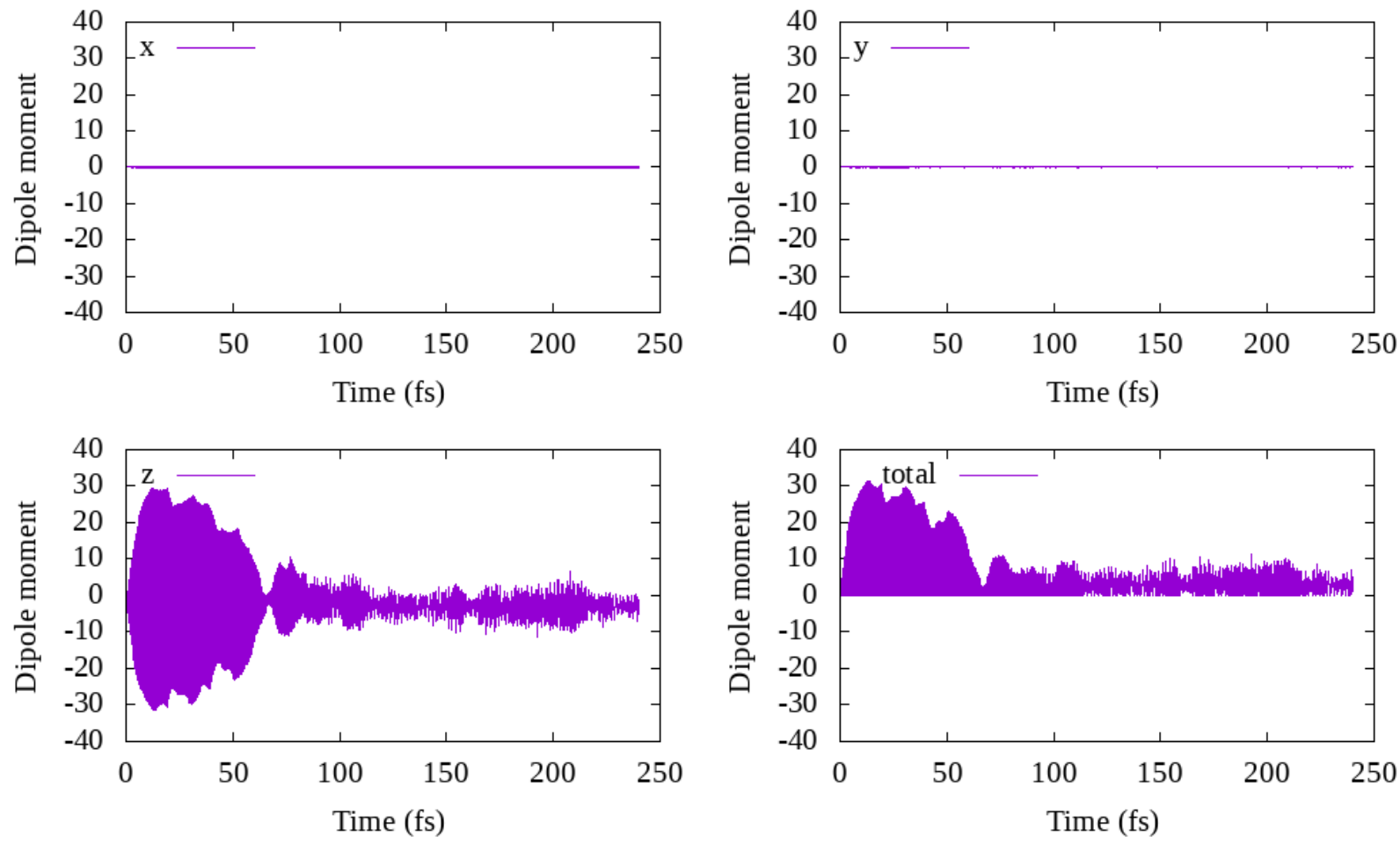

Figure S9. Variation of different components of the dipole moment (Debye) from the application of a 0.001 au trapezoid electric field along z-direction in $\left[\mathrm{Al}_{13} \mathrm{~N}_{2}\right]^{-1}$ with $\mathrm{C}_{2 \mathrm{v}}$ symmetry. 

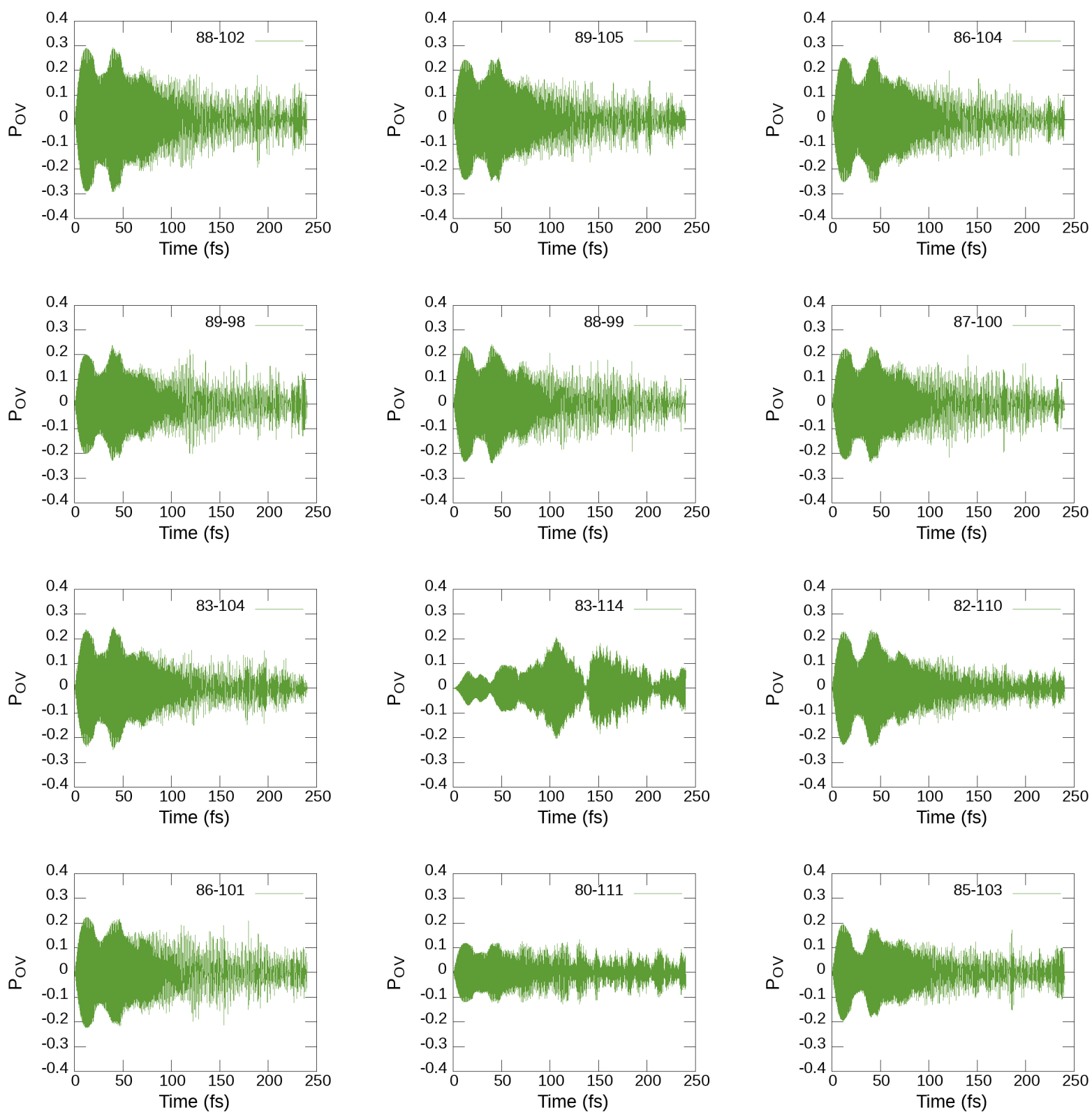

a) 

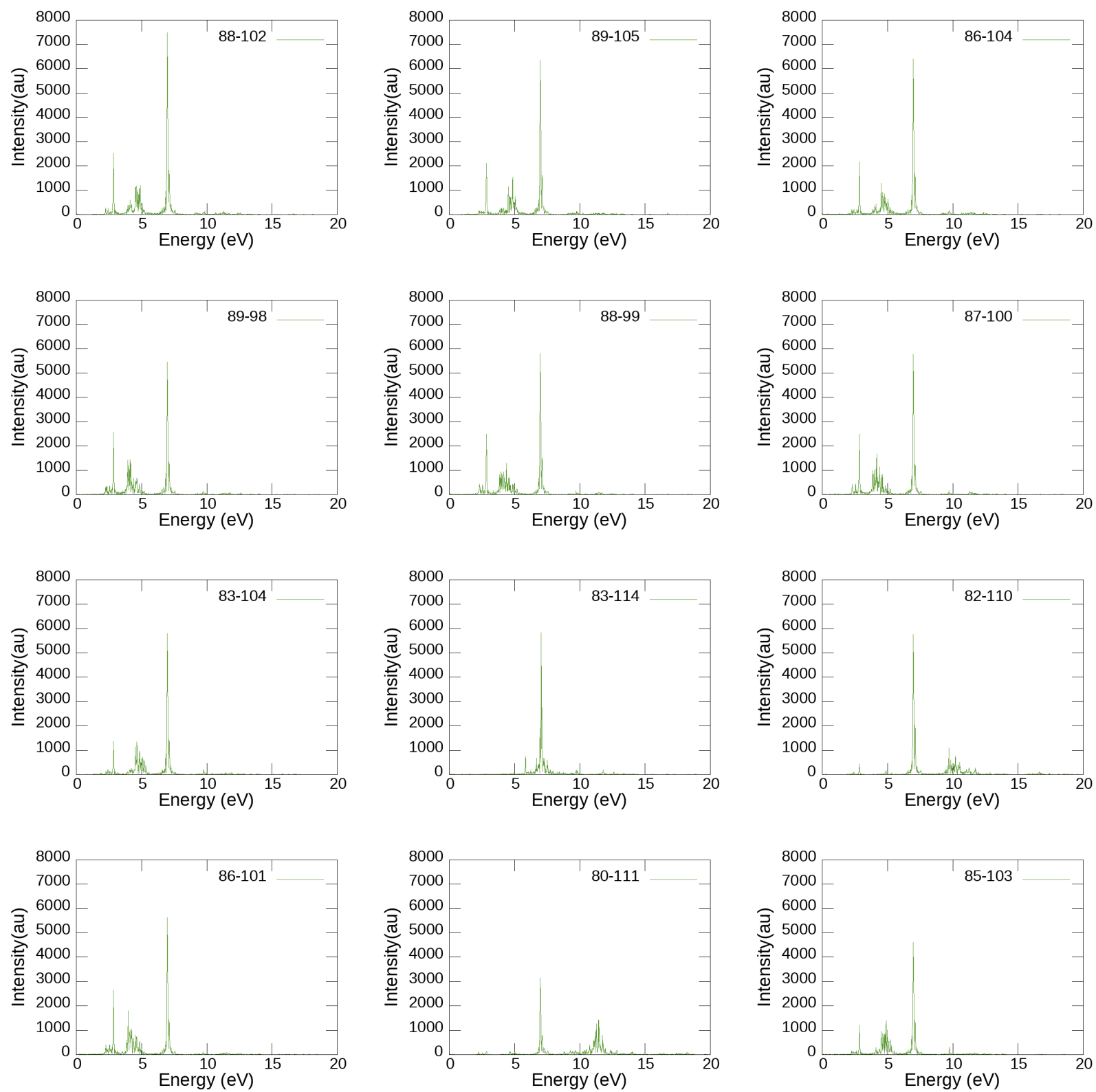

b) 

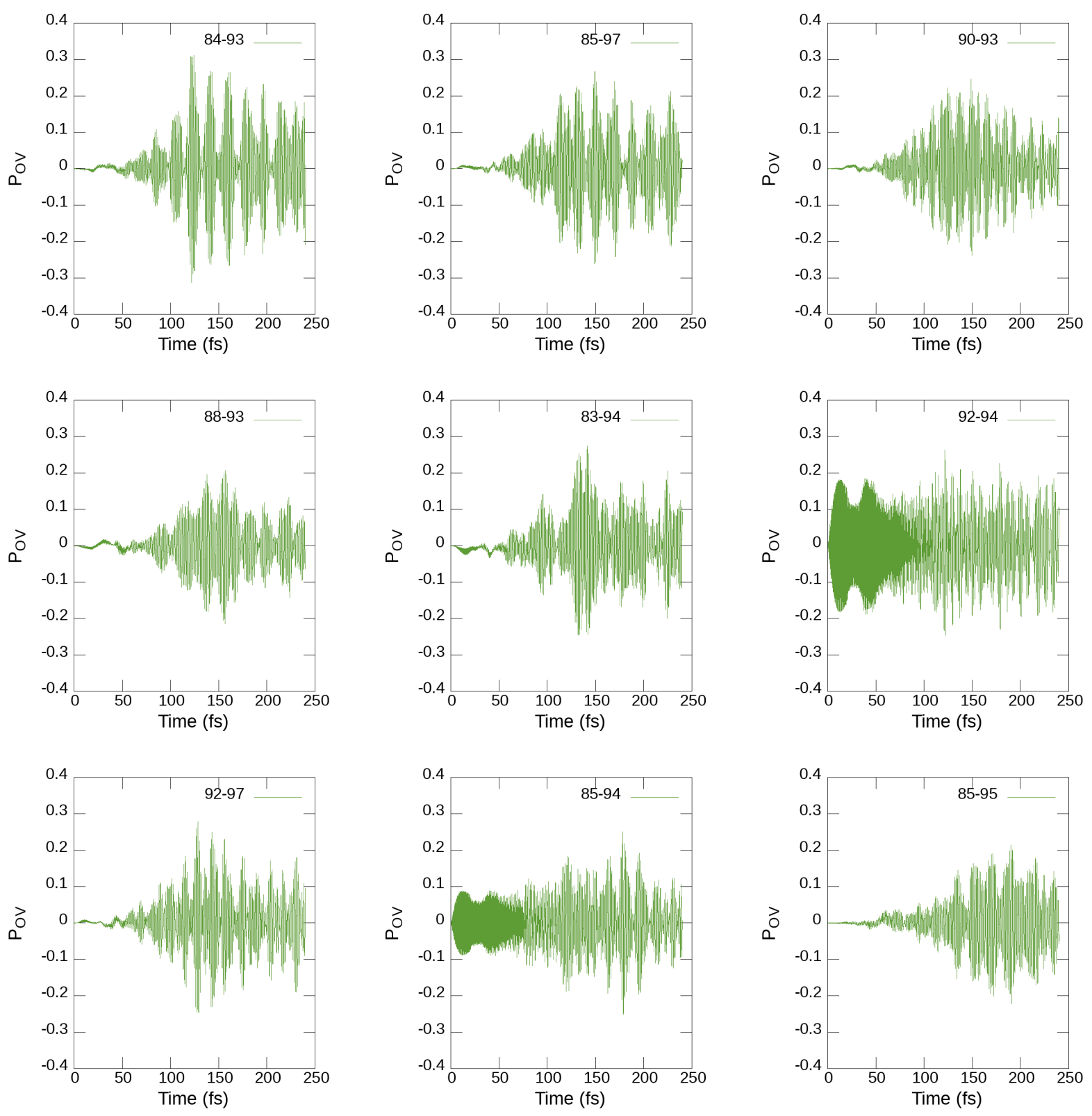

C) 

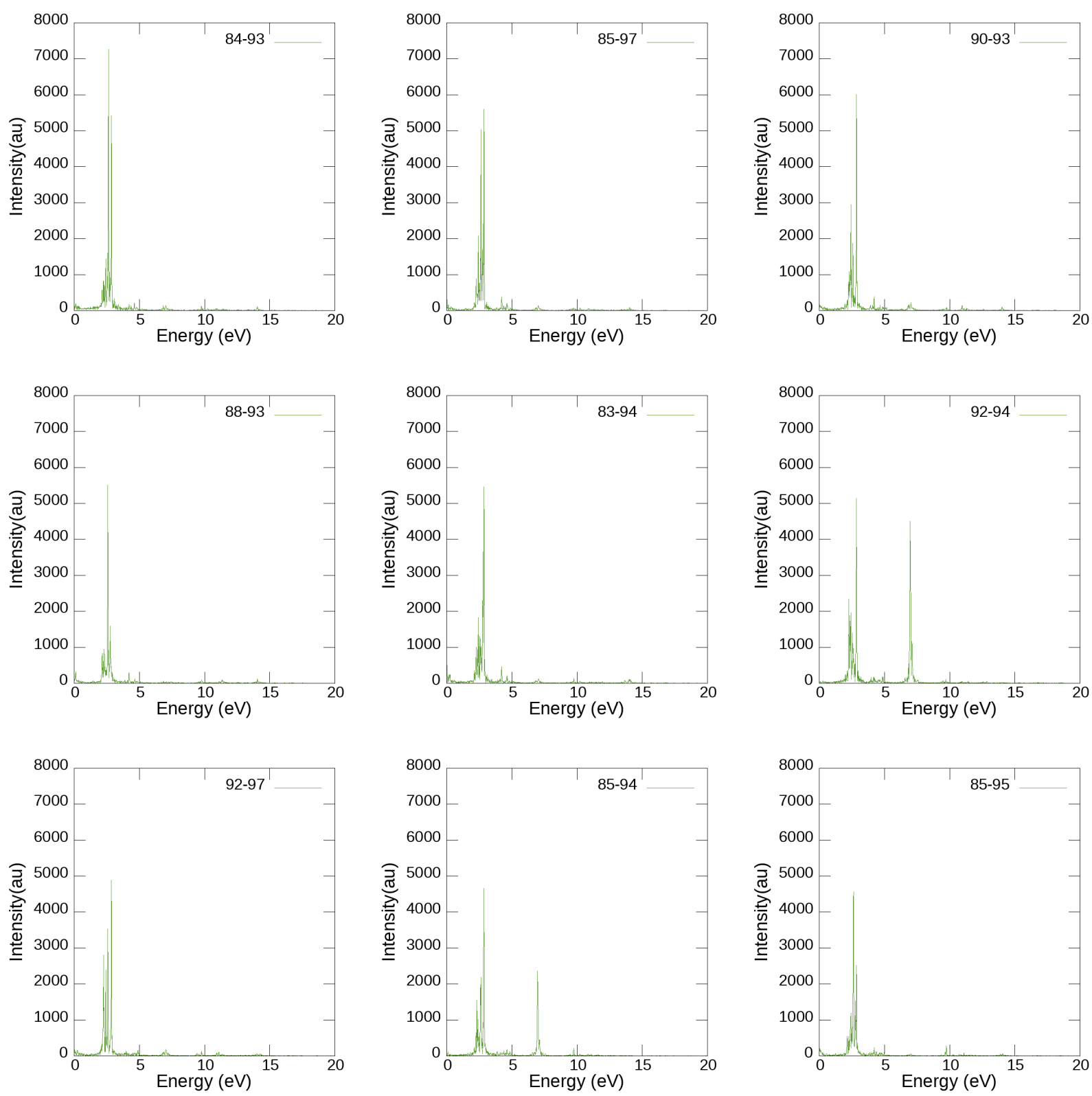

Figure S10. $P_{O V}$ for different transitions that contribute the major peaks at $7 \mathrm{eV}$ (a), and the FT peaks for the corresponding transitions (b). Pov for different transitions that contribute the major peaks at $2 \mathrm{eV}-3 \mathrm{eV}$ (c) and the FT peaks for the corresponding transitions (d). These are obtained from the application of a 0.001 au trapezoid field with a frequency corresponding to $7 \mathrm{eV}$ along the z-direction in the $\left[\mathrm{Al}_{13} \mathrm{~N}_{2}\right]^{-1}$ system. 

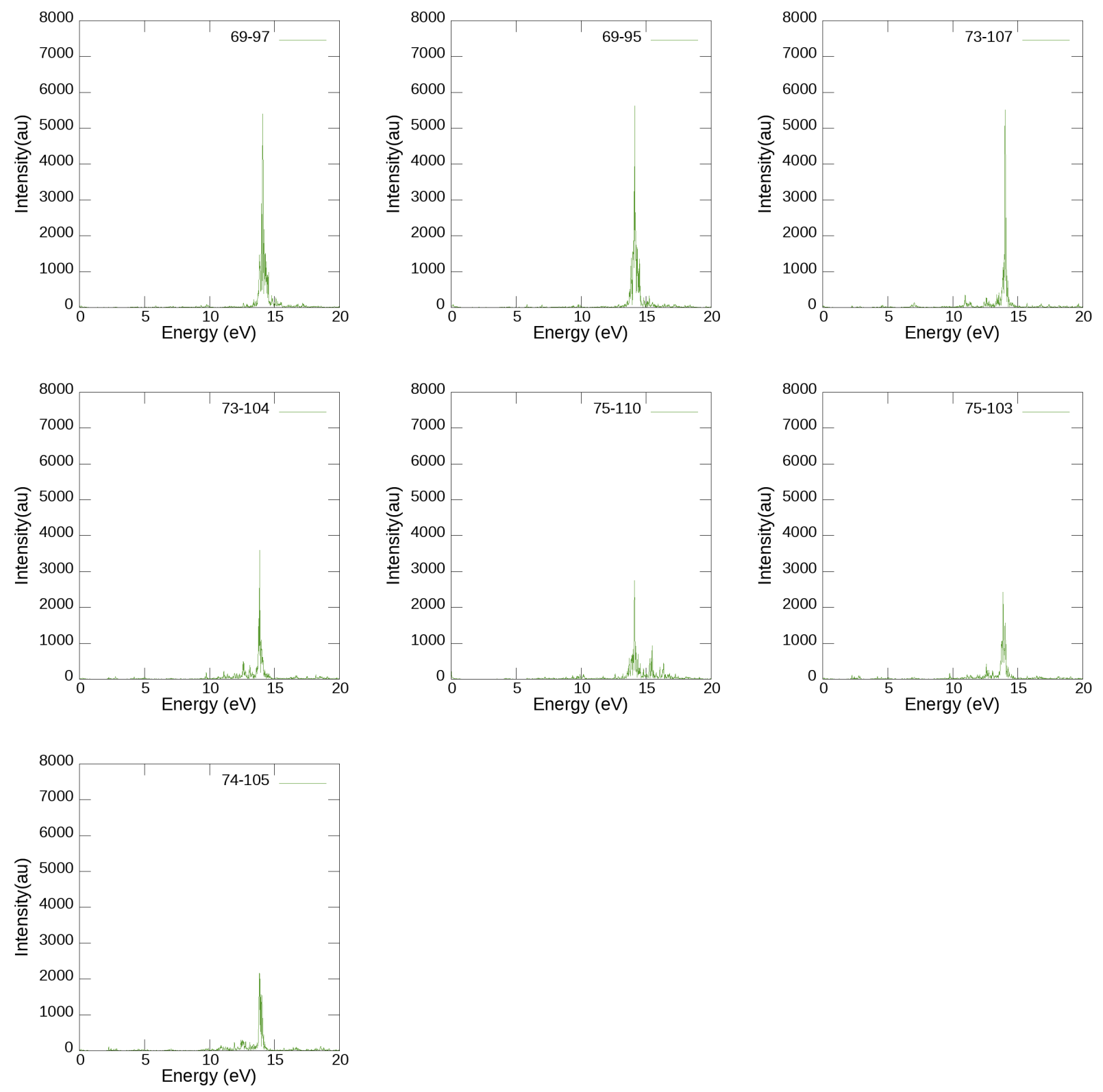

a) 

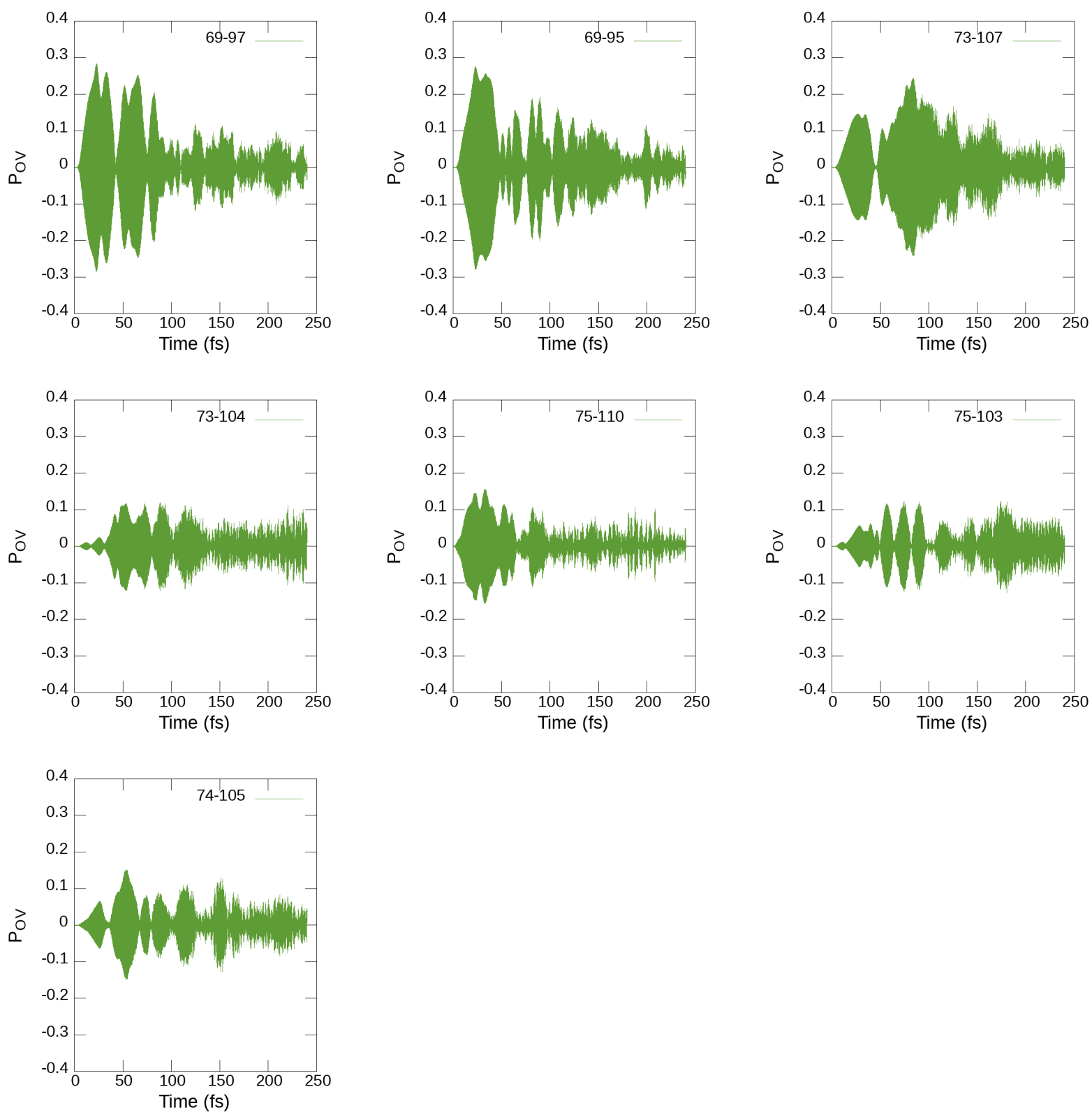

b)

Figure S11. FT peaks (a) and the $\mathrm{P}_{\mathrm{OV}}(\mathrm{b})$ for the transitions that give two-photon absorption peaks. These are obtained from the application of a 0.001 au trapezoid field with a frequency corresponding to $7 \mathrm{eV}$ along the $\mathrm{z}$-direction in the $\left[\mathrm{Al}_{13} \mathrm{~N}_{2}\right]^{-1}$ system. 

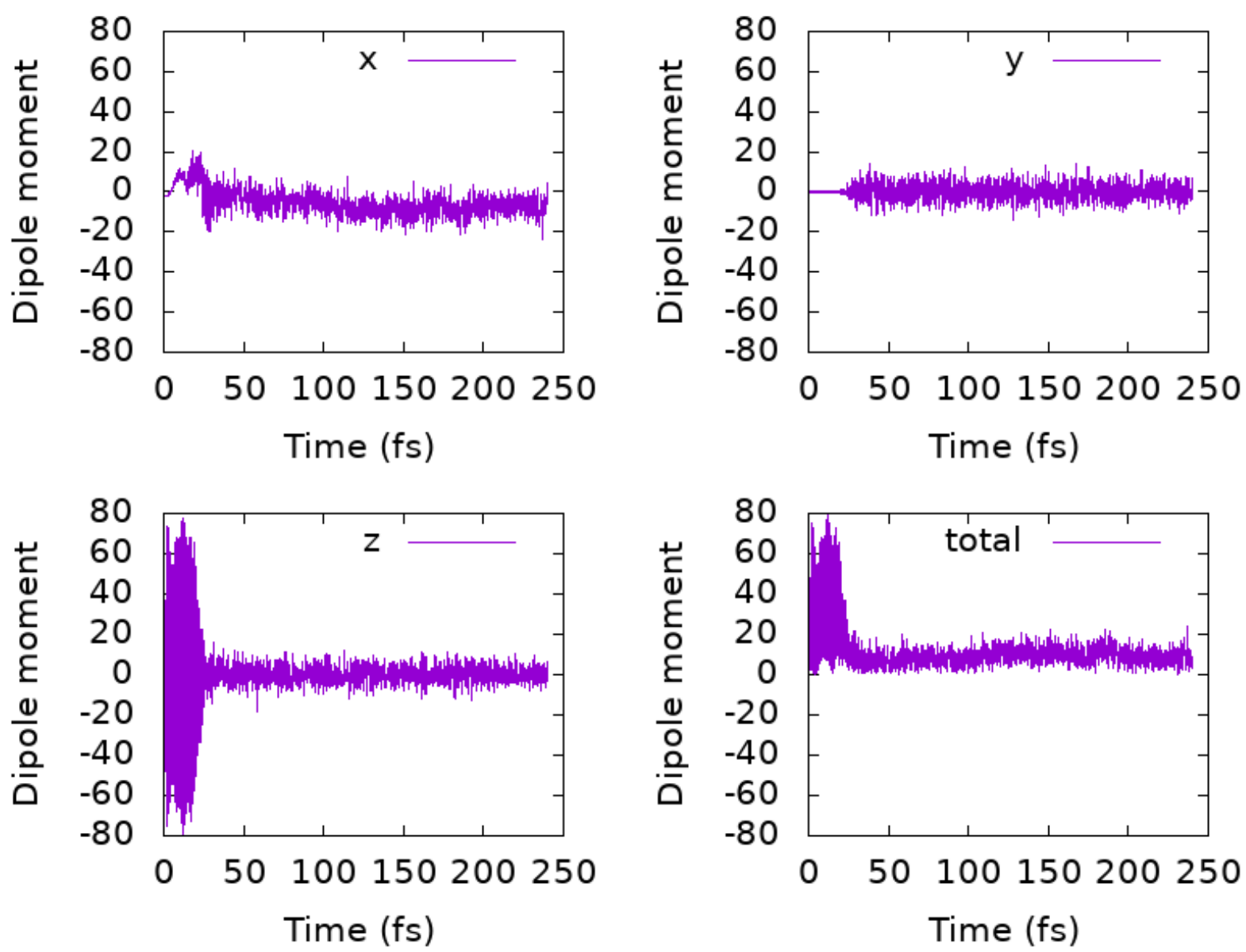

Figure S12. Variation of different components of the dipole moment (Debye) from the application of the $7 \mathrm{eV}$ trapezoid field of 0.01 au along the z-direction of the $\left[\mathrm{Al}_{13} \mathrm{~N}_{2}\right]^{-1}$ system. 

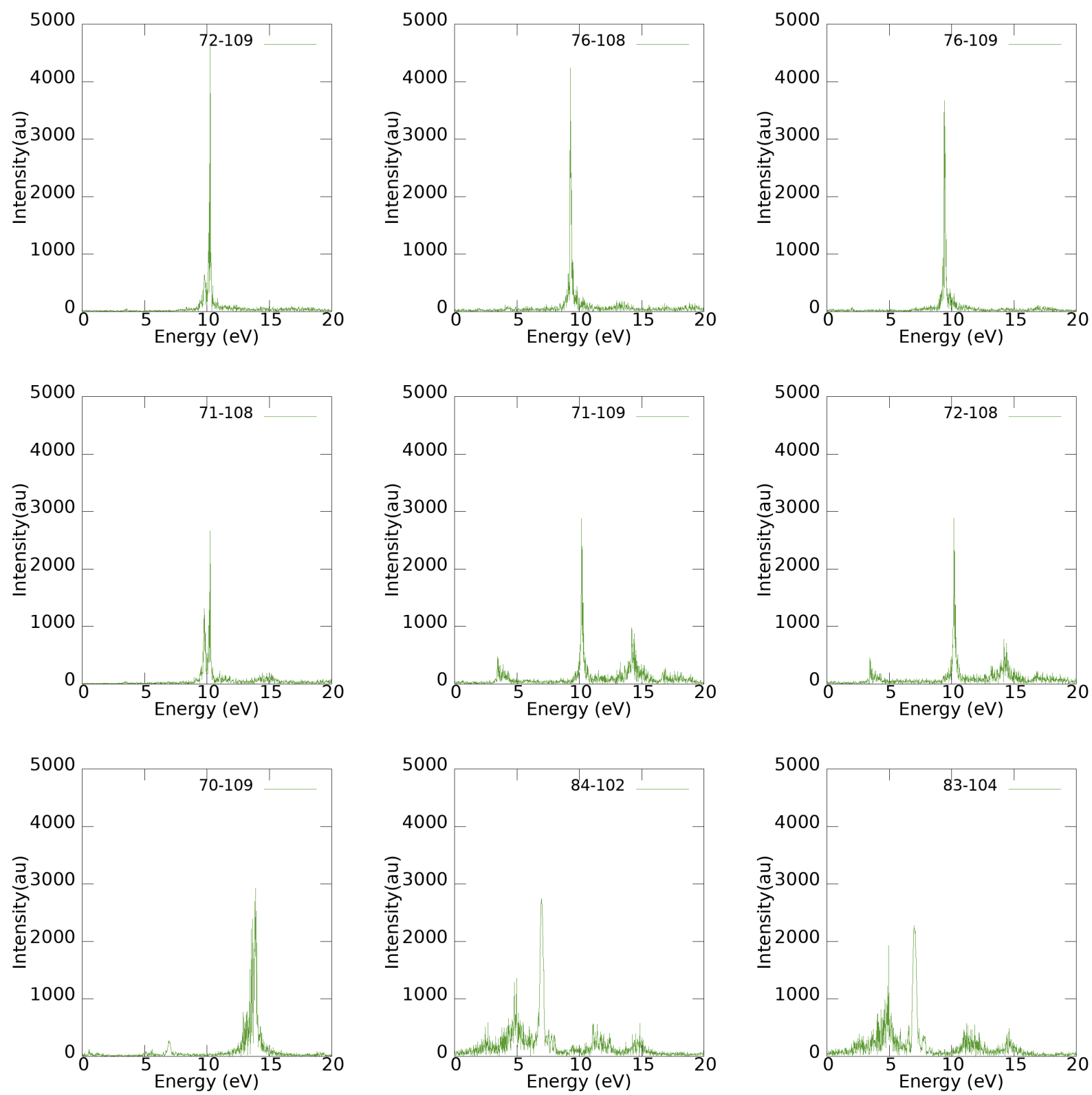

a) 

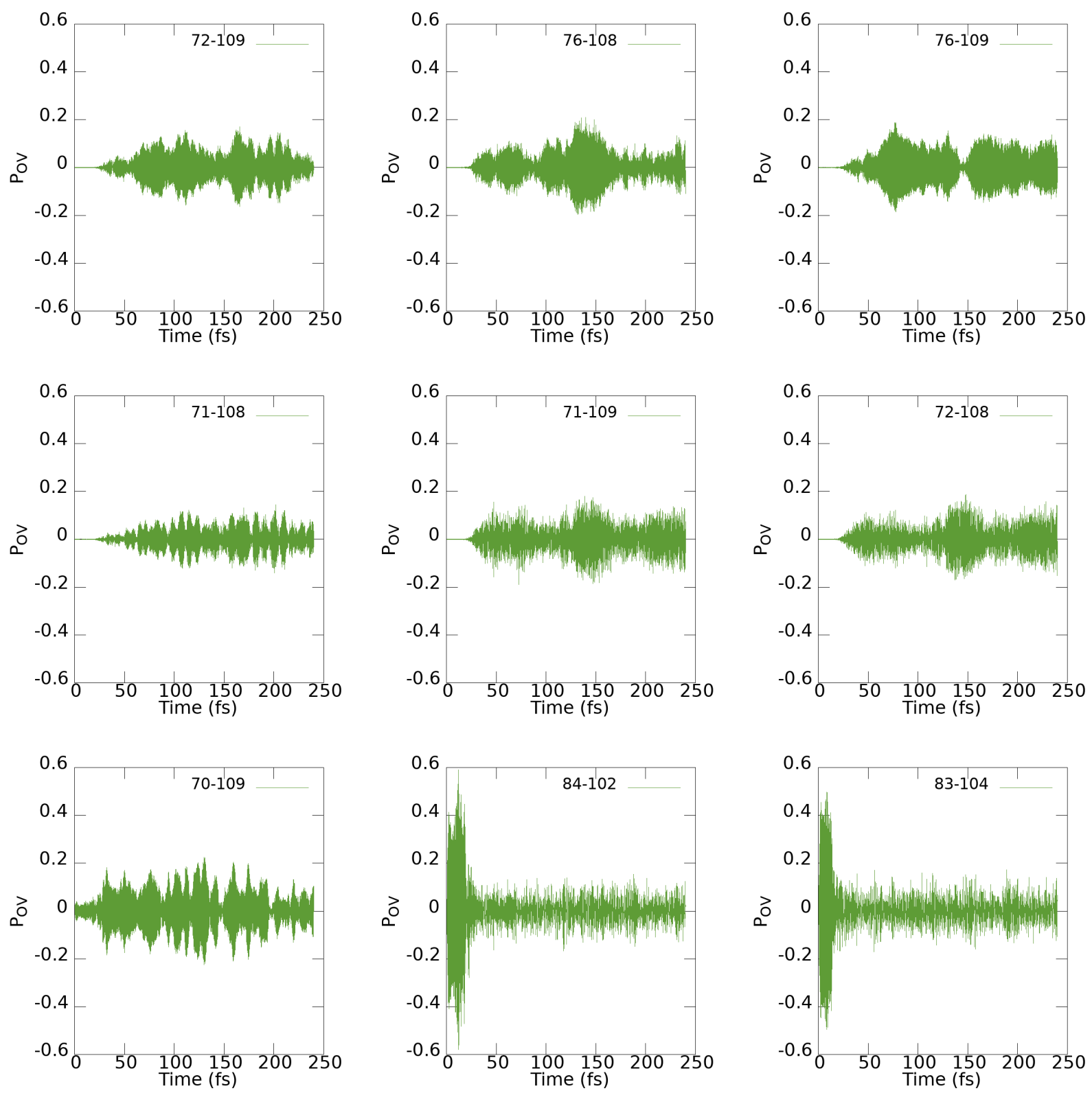

b)

Figure S13. (a) Highest intensity Fourier transformed peaks and (b) the $P_{O V}$ elements for different single-particle transitions when a 0.01 au trapezoid wave electric field of $7 \mathrm{eV}$ is applied along the z-direction in $\left[\mathrm{Al}_{13} \mathrm{~N}_{2}\right]^{-1}$. 

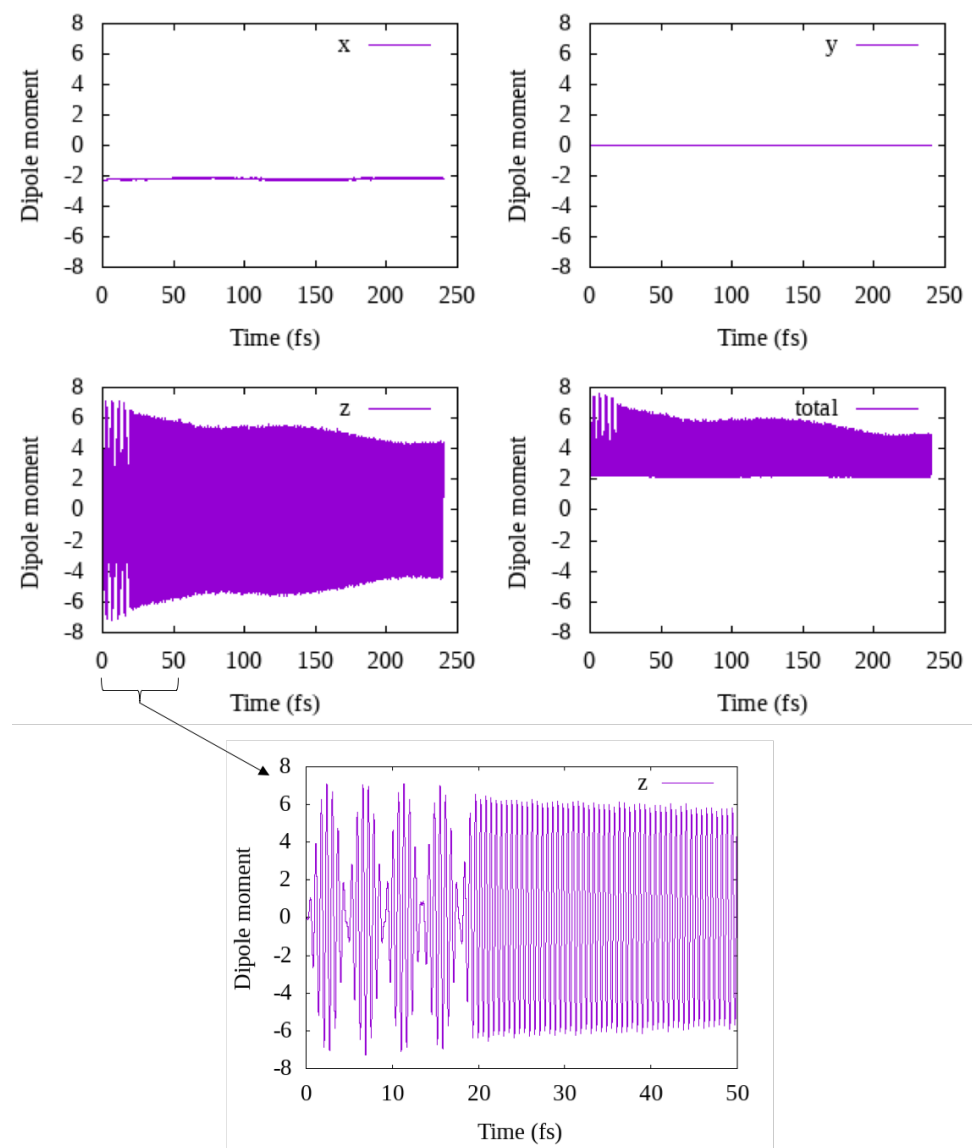

Figure S14. Variation of different components of the dipole moment (Debye) from the application of a $6 \mathrm{eV}$ trapezoid field with a field strength of 0.001 au applied along the $\mathrm{z}$ direction of the $\left[\mathrm{Al}_{13} \mathrm{~N}_{2}\right]^{-1}$ system. 

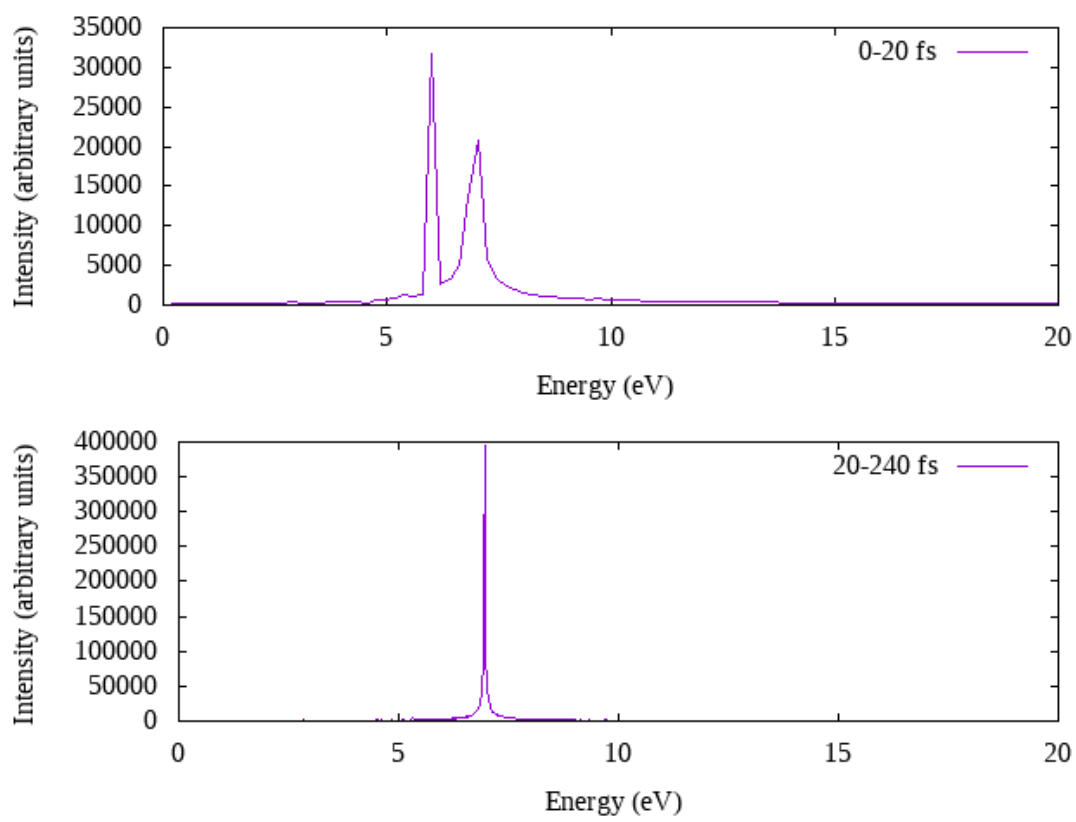

Figure S15. Fourier transform of the dipole moment (Debye) on application of a 0.001 au zpolarized $6 \mathrm{eV}$ trapezoidal field along the $\mathrm{z}$-direction in the $\left[\mathrm{Al}_{13} \mathrm{~N}_{2}\right]^{-1}$ system.
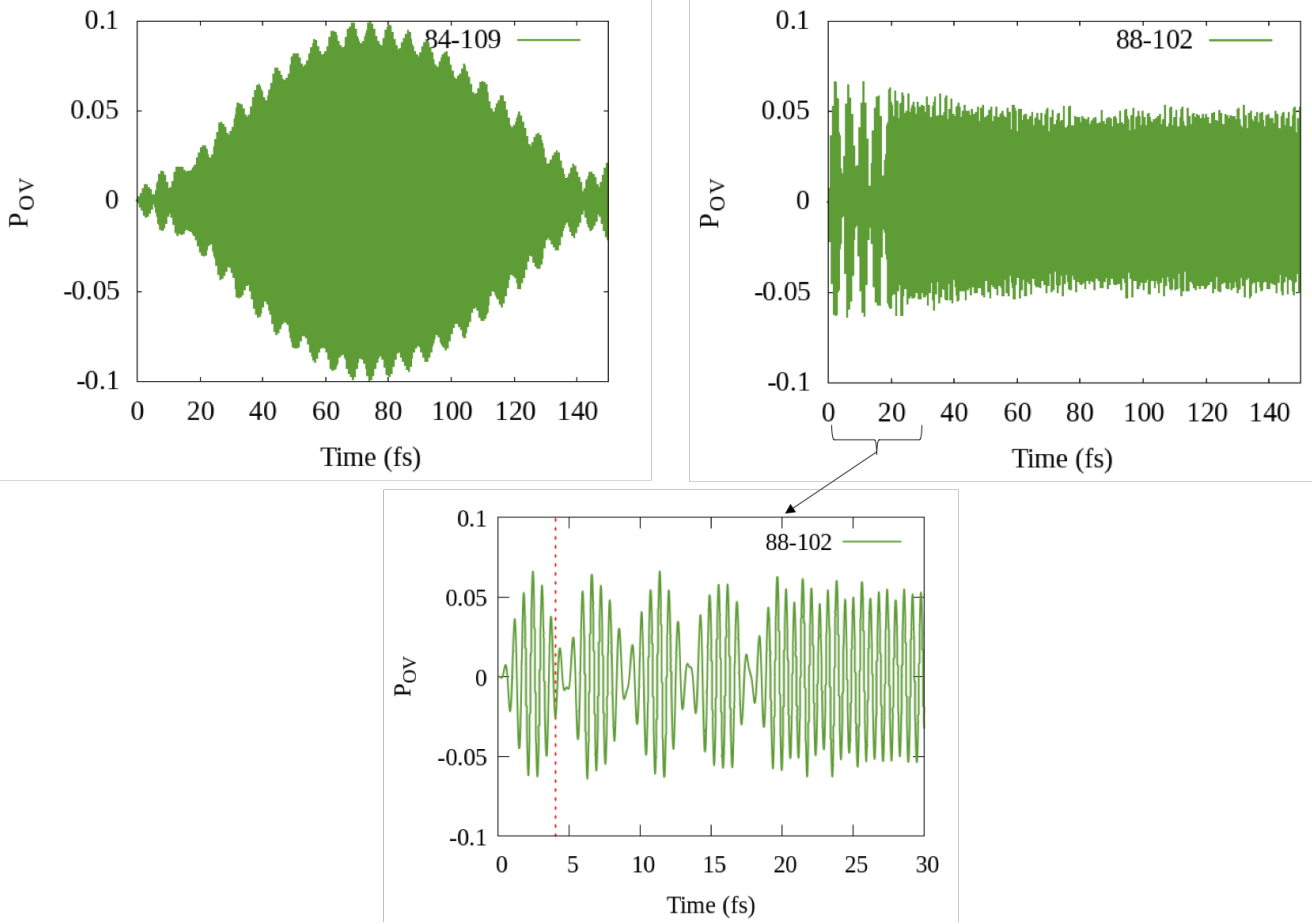

Figure S16. POV for the transitions that give the highest intensity FT peaks when a 0.001 au trapezoid field electric field of $6 \mathrm{eV}$ is applied along the z-direction to $\left[\mathrm{Al}_{13} \mathrm{~N}_{2}\right]^{-1}$. 

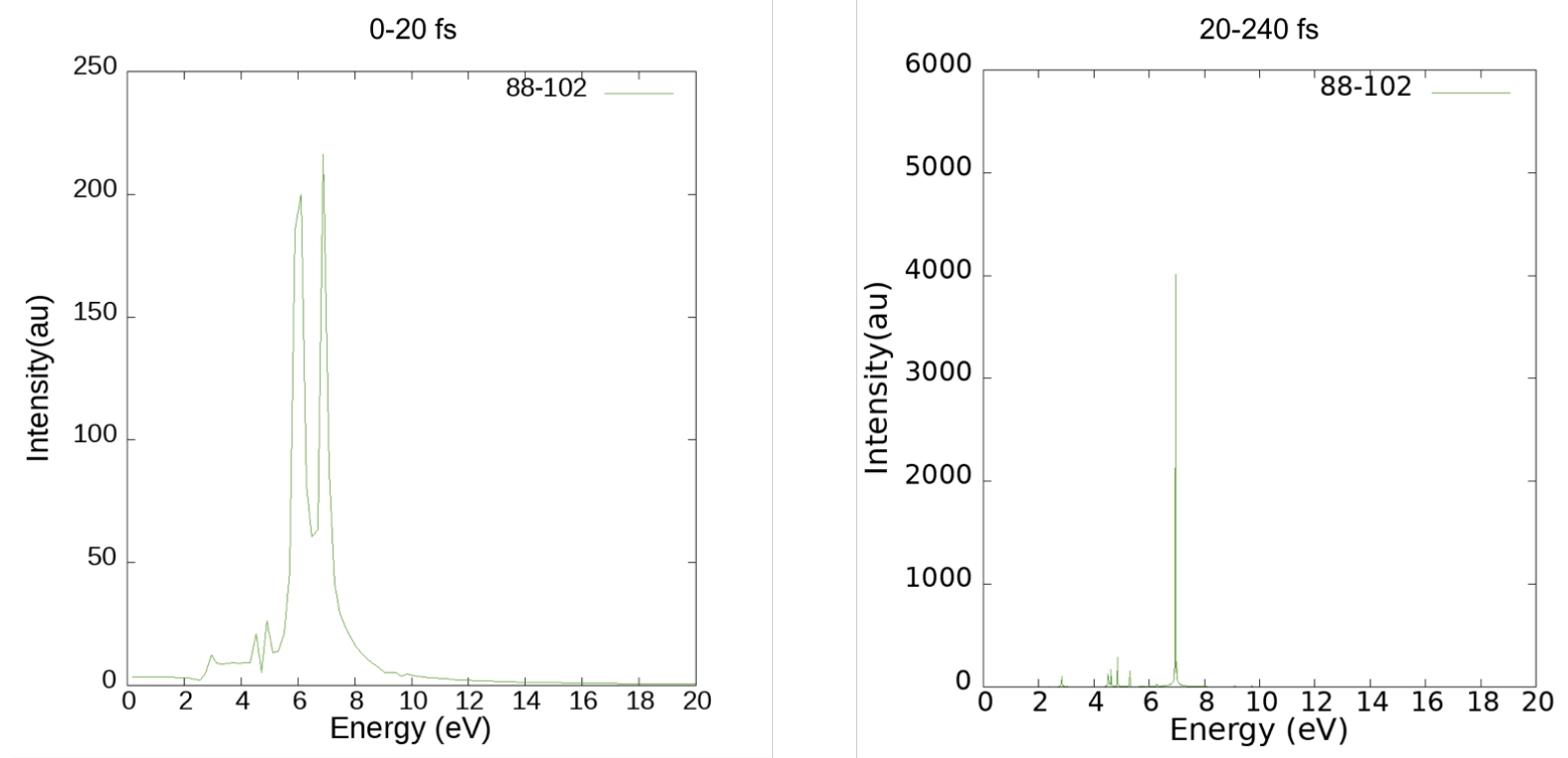

Figure S17. Fourier transformed peaks for the transition $88 \rightarrow 102$ during and after the application of the applied laser field. The FT after the application of the field does not show the peak at $6 \mathrm{eV}$ which was present when the Fourier transform was taken during the application of laser field. These FTs are obtained from the application of a 0.001 au trapezoid field with frequency corresponding to $6 \mathrm{eV}$ along the z-direction in $\left[\mathrm{Al}_{13} \mathrm{~N}_{2}\right]^{-1}$.
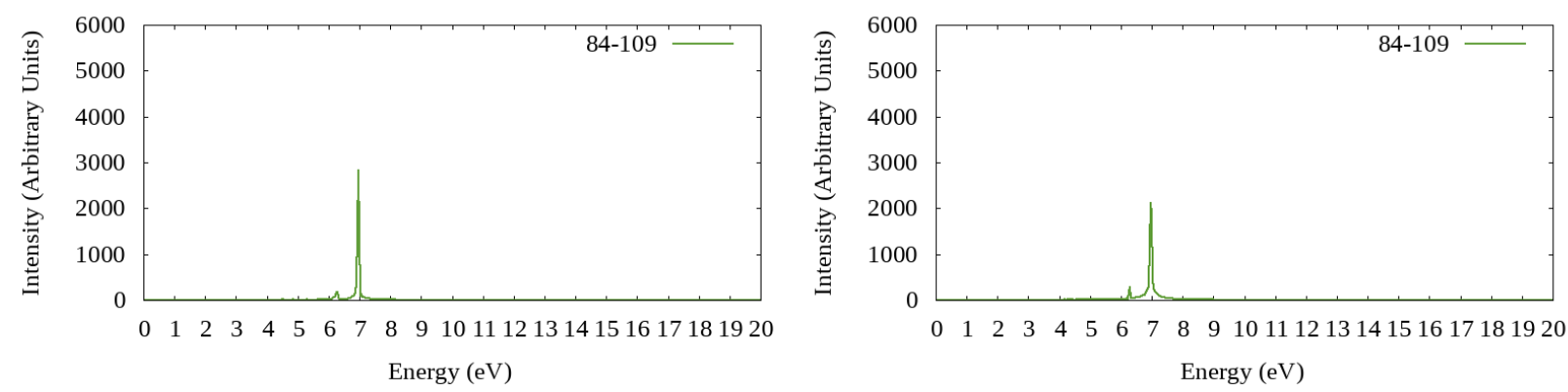

Figure S18. Fourier transformed peaks for the $84 \rightarrow 109$ transition from 21-130 fs (left) and from 130-240 fs (right). These are obtained after the application of a 0.001 au trapezoid field with a frequency corresponding to $6 \mathrm{eV}$ along the z-direction in $\left[\mathrm{Al}_{13} \mathrm{~N}_{2}\right]^{-1}$. 

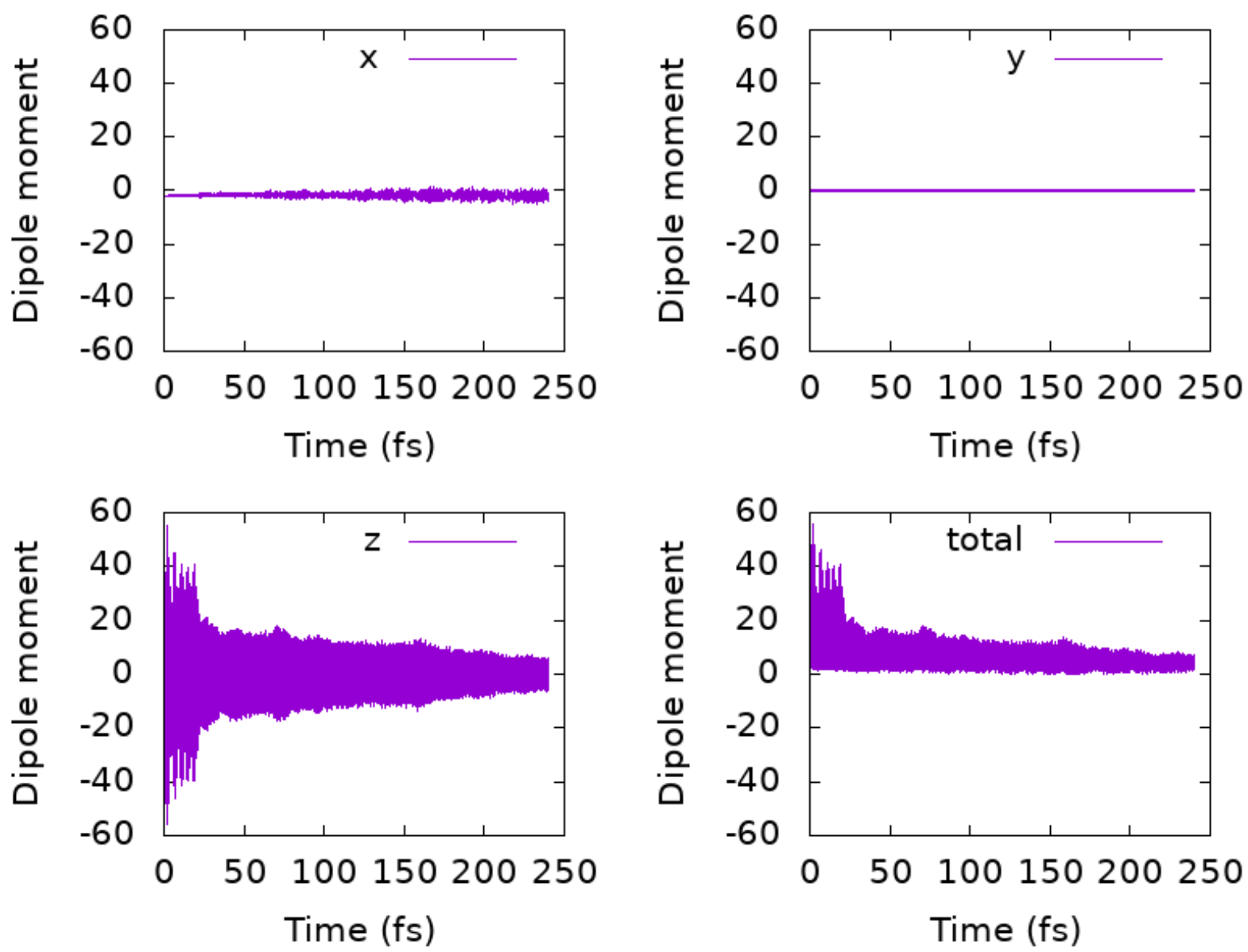

Figure S19. Variation of different components of the dipole moment (Debye) upon the application of a $6 \mathrm{eV}$ trapezoid field with a field strength of 0.01 au along the z-direction of $\left[\mathrm{Al}_{13} \mathrm{~N}_{2}\right]^{-1}$. 

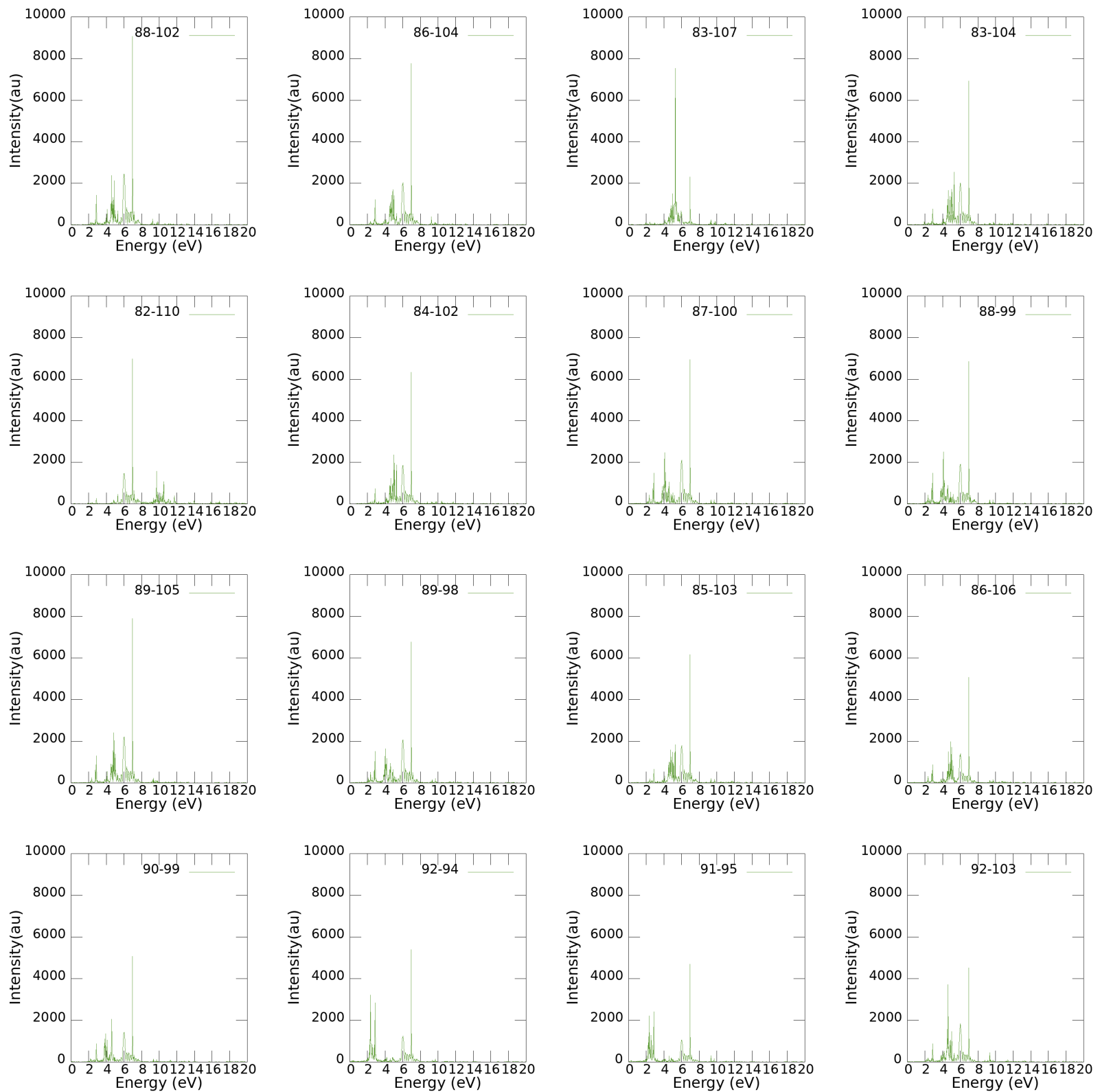

a) 

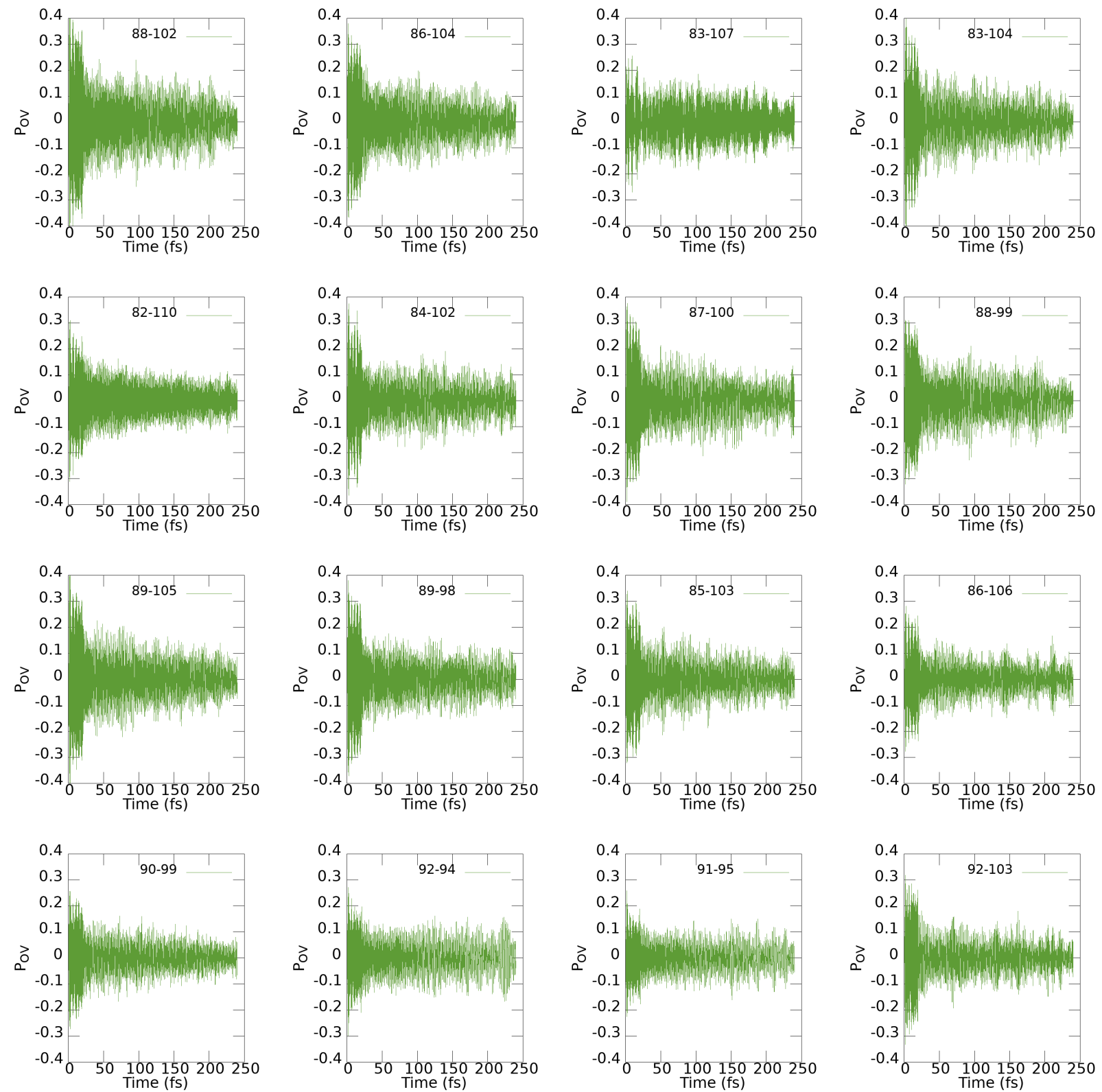

b)

Figure S20. (a) Highest intensity Fourier transformed peaks and (b) Pov elements when a 0.01 au trapezoid electric field with a frequency of $6 \mathrm{eV}$ is applied along the z-direction in $\left[\mathrm{Al}_{13} \mathrm{~N}_{2}\right]^{-1}$. 

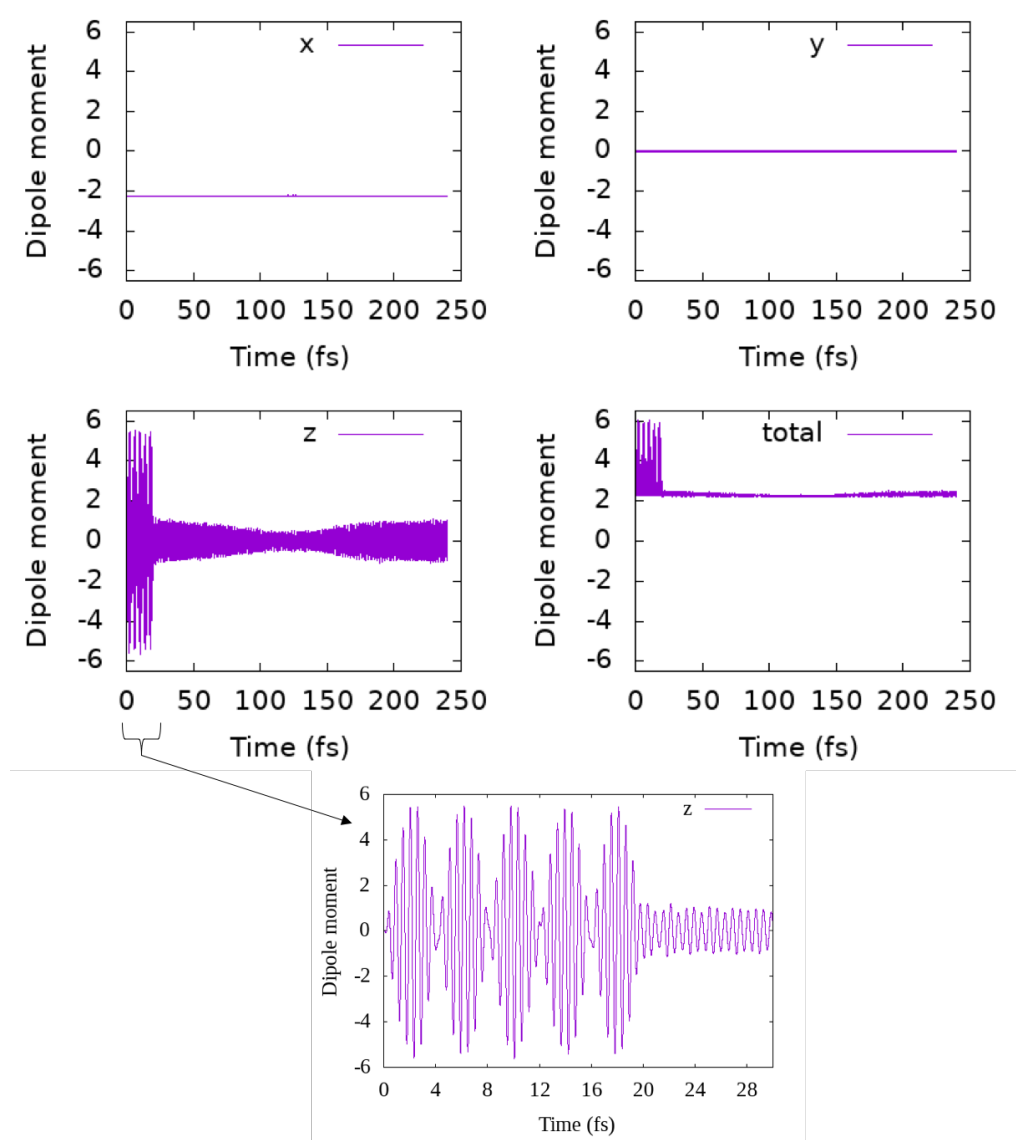

a)
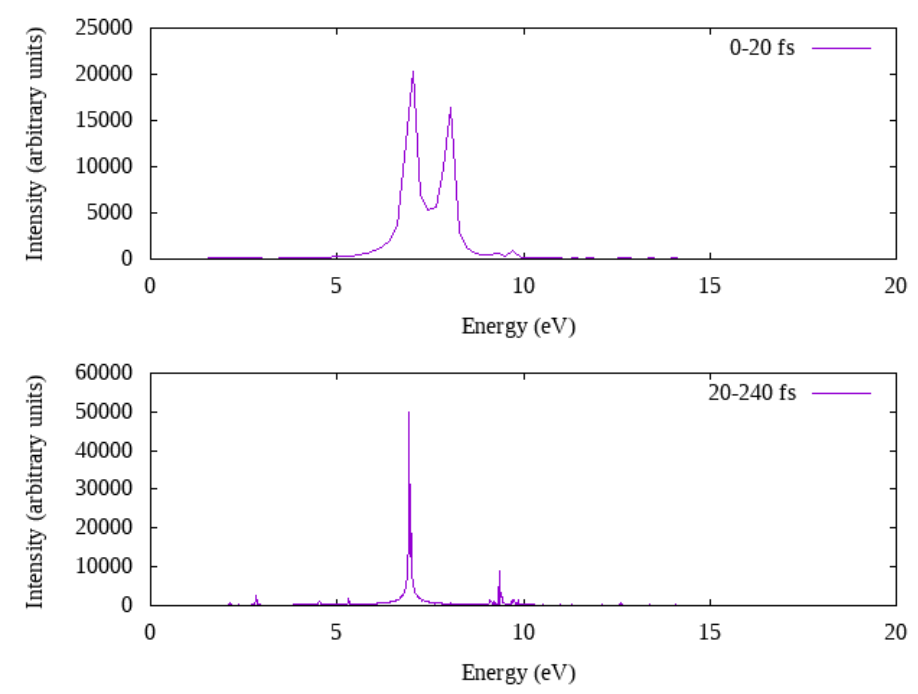

b)

Figure S21. (a) Variation of different components of the dipole moment (Debye) from the application of a $8 \mathrm{eV}$ trapezoid field with a field strength of 0.001 au along the $z$-direction of the $\left[\mathrm{Al}_{13} \mathrm{~N}_{2}\right]^{-1}$ system. (b) Fourier transform of the dipole moment (Debye) on application of a 0.001 au z-polarized $8 \mathrm{eV}$ trapezoidal field along the $\mathrm{z}$-direction in the $\left[\mathrm{Al}_{13} \mathrm{~N}_{2}\right]^{-1}$ system. 

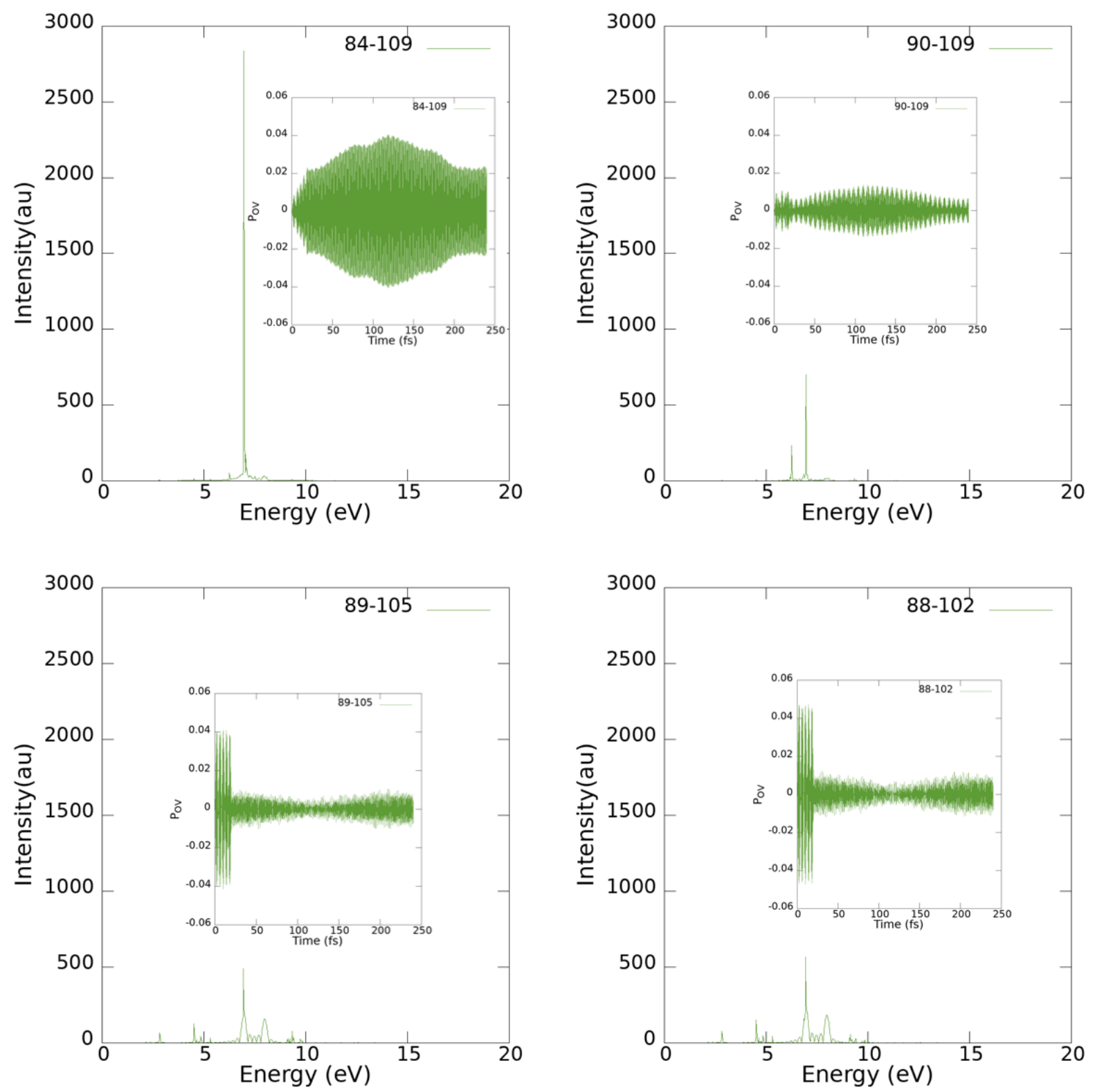

a) 

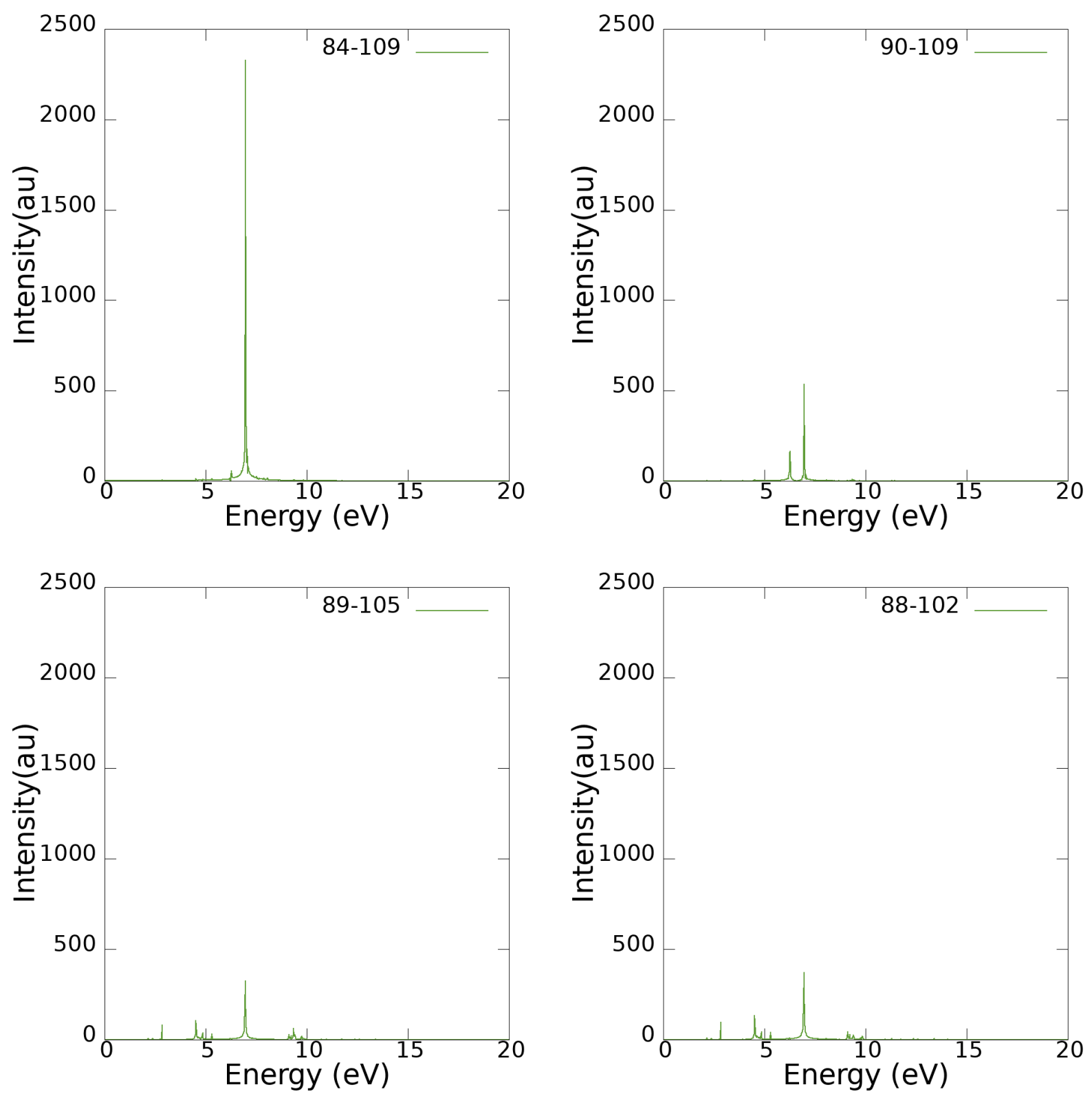

b) 

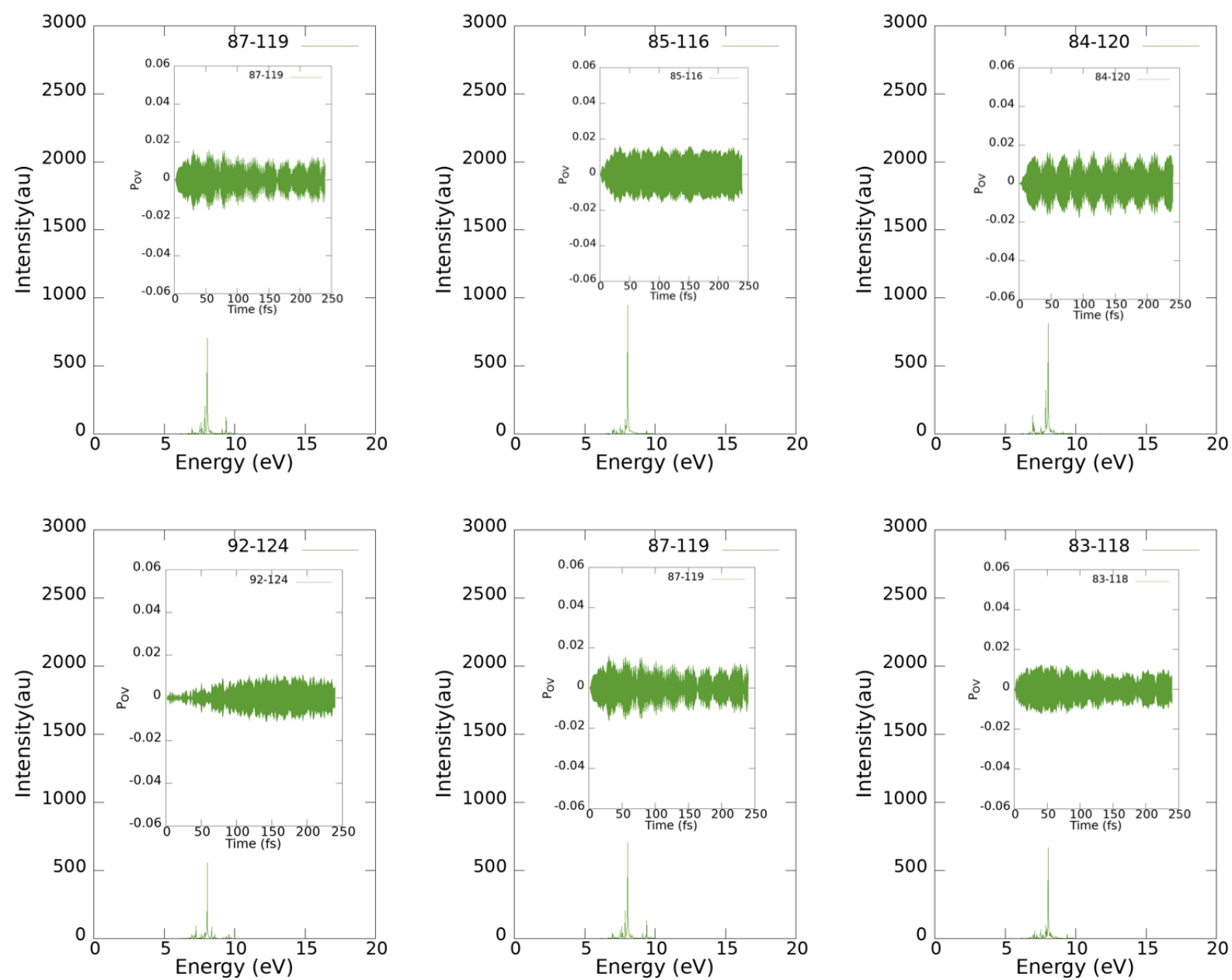

c)

Figure S22. (a) Transitions that give highest intensity FT peaks at $7 \mathrm{eV}$ and the Pov for the corresponding transitions as insets. The FT are obtained with Pov dynamics from 0-240 fs. (b) FT peaks obtained by only considering $P_{\mathrm{OV}}$ dynamics after $20 \mathrm{fs}$ for the transitions that give the $7 \mathrm{eV}$ peak. (c) Transitions that give the highest intensity FT peaks at $8 \mathrm{eV}$ and the Pov for the corresponding transitions as insets. These are obtained from the application of a 0.001 au trapezoid field with a frequency corresponding to $8 \mathrm{eV}$ along the $\mathrm{z}$-direction in $\left[\mathrm{Al}_{13} \mathrm{~N}_{2}\right]^{-1}$. 

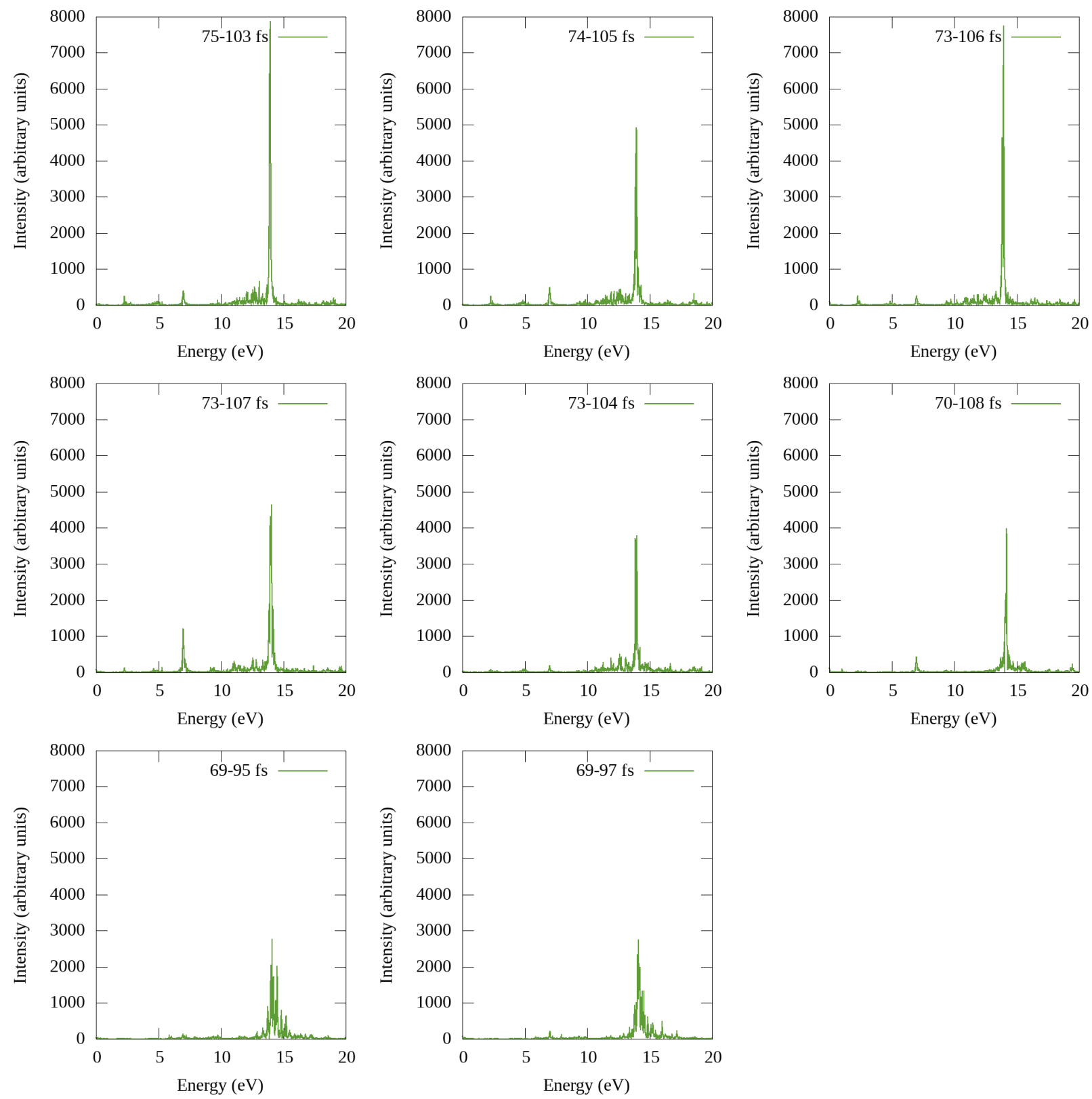

a) 

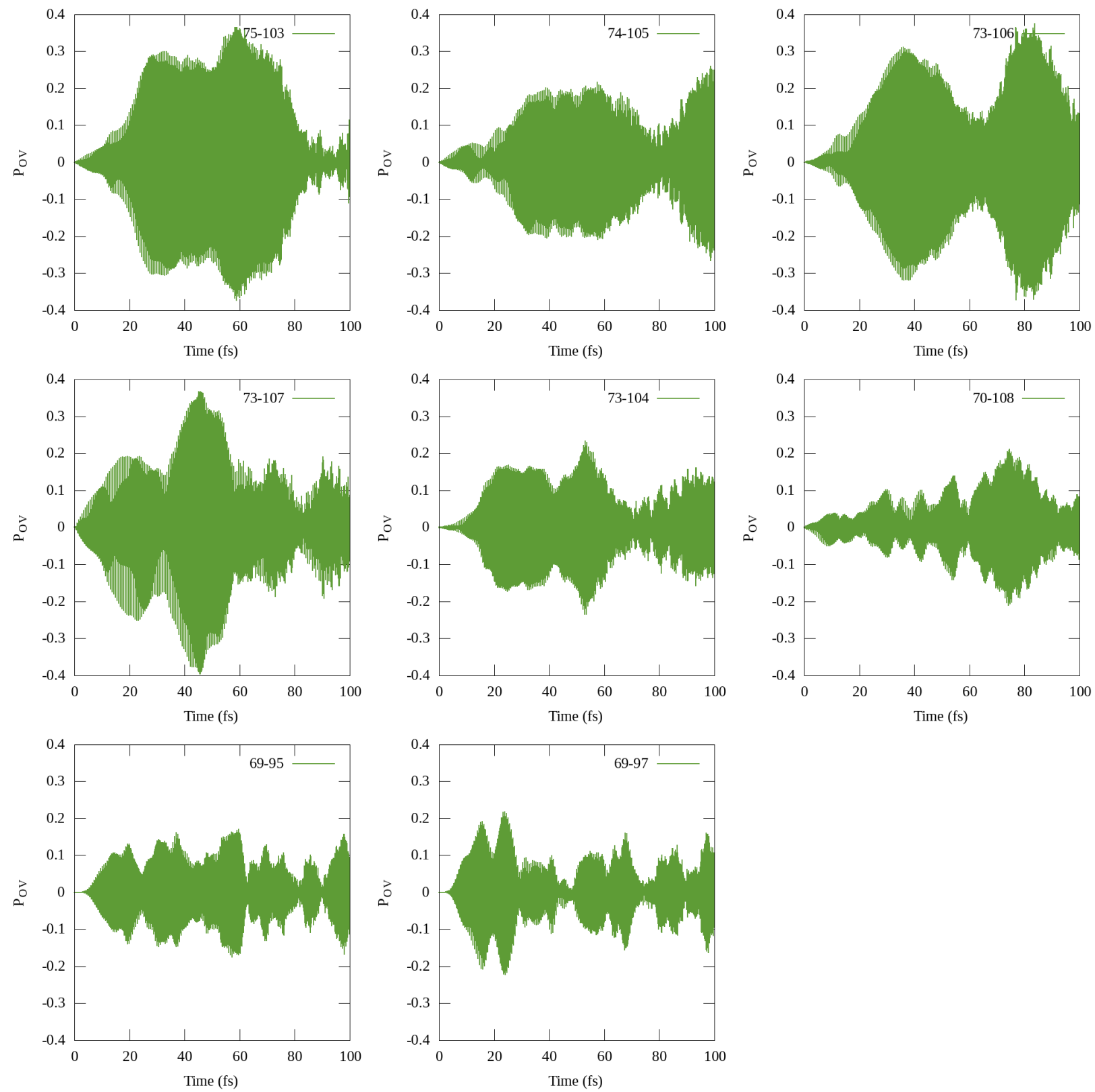

b)

Figure S23. FT peaks (a) and the $\mathrm{P}_{\mathrm{OV}}(\mathrm{b})$ for the transitions that give two-photon absorption peaks. These are obtained from the application of a 0.001 au trapezoid field with a frequency corresponding to $7 \mathrm{eV}$ along the $\mathrm{x}$-direction in the $\left[\mathrm{Al}_{13} \mathrm{~N}_{2}\right]^{-1}$ system. 